\title{
Classical Cepheid Pulsation Models. X. The Period-Age Relation
}

\author{
G. Bono ${ }^{1}$, M. Marconi ${ }^{2}$, S. Cassisi ${ }^{3}$, F. Caputo ${ }^{1}$, W. Gieren ${ }^{4}$, G. Pietrzynski ${ }^{4,5}$
}

\begin{abstract}
We present new Period-Age (PA) and Period-Age-Color (PAC) relations for fundamental and first overtone classical Cepheids. Current predictions rely on homogeneous sets of evolutionary and pulsation models covering a broad range of stellar masses and chemical compositions. We found that PA and PAC relations present a mild dependence upon metal content. Moreover, the use of different PA and PAC relation for fundamental and first overtone Cepheids improves the accuracy of age estimates in the short-period $(\log P<1)$ range (old Cepheids), because they present smaller intrinsic dispersions. At the same time, the use of the $\mathrm{PAC}$ relations improves the accuracy in the long-period $(\log P \geq 1$ ) range (young Cepheids), since they account for the position of individual objects inside the instability strip.

We performed a detailed comparison between evolutionary and pulsation ages for a sizable sample of LMC (15) and SMC (12) clusters which host at least two Cepheids. In order to avoid deceptive uncertainties in the photometric absolute zero-point, we adopted the homogeneous set of B,V,I data for clusters and Cepheids collected by OGLE. We also adopted the same reddening scale. The different age estimates agree at the level of $20 \%$ for LMC clusters and of $10 \%$ for SMC clusters. We also performed the same comparison for two Galactic clusters (NGC6067, NGC7790) and the difference in age is smaller than $20 \%$.

These findings support the use of PA and PAC relations to supply accurate estimates of individual stellar ages in the Galaxy and in external Galaxies. The main advantage of this approach is its independence from the distance.
\end{abstract}

\footnotetext{
${ }^{1}$ INAF - Osservatorio Astronomico di Roma, via Frascati 33, 00040 Monte Porzio Catone, Italy; bono@mporzio.astro.it, caputo@mporzio.astro.it

${ }^{2}$ INAF - Osservatorio Astronomico di Capodimonte, via Moiariello 16, 80131 Napoli, Italy; marcella@na.astro.it

${ }^{3}$ INAF - Osservatorio Astronomico di Teramo, via M. Maggini, 64100 Teramo, Italy; cassisi@te.astro.it

${ }^{4}$ Universidad de Concepcion, Departamento de Fisica, Astronomy Group, Casilla 160-C, Concepcion, Chile; wgieren@coma.cfm.udec.cl, pietrzyn@hubble.cfm.udec.cl

${ }^{5}$ Warsaw University Observatory, Al. Ujazdowskie 4, 00-478 Warszawa, Poland
} 
Subject headings: Cepheids - Galaxy: stellar content - hydrodynamics - stars: evolution - stars: oscillations

\section{Introduction}

Classical Cepheids are crucial objects in several long-standing astrophysical problems. These variable stars, due to their intrinsic luminosity and regular luminosity variation, can easily be detected in the outskirt of the Galactic disk and in external galaxies. Cepheids are the most popular primary distance indicators, since the pulsation periods and the colors are connected with the luminosity, and therefore the Period-Luminosity (PL) and the PeriodLuminosity-Color (PLC) relation can be adopted for estimating cosmic distances (Feast 1999; Udalski et al. 1999a,b,c; Freedman et al. 2001; Saha et al. 2001; Fouqué et al. 2003; Tammann, Sandage, \& Reindl 2003; Storm et al. 2004).

Classical Cepheids cover the whole range of intermediate-mass stars, and thus it is not surprising that these objects are typically adopted to trace Population I stars in the Galactic disk (Kraft \& Schmidt 1963), and star-forming regions in extragalactic systems (Elmegreen \& Efremov 1996). The use of Cepheids as tracers of young stellar populations was supplemented by the evidence that, if these objects obey to a PL relation, they also obey to a Period-Age (PA) relation. Therefore, by taking into account the Mass-Luminosity relation and the stellar ages predicted by evolutionary models, it was soon recognized (Kippenhahn \& Smith 1969; Meyer-Hofmeister 1969) that an increase in the pulsation period implies an increase in the stellar mass, and in turn a decrease in the Cepheid age. On the basis of these plane physical arguments Efremov (1978) derived an empirical PA relation by adopting Cepheids in Galactic, in M31, and in Large Magellanic Cloud (LMC) clusters, whose age was independently estimated (see also Tsvetkov 1989). More recently, Magnier et al. (1997, hereinafter M97) derived a new semi-empirical PA relation and used Cepheids in NGC206, the super-association in M31, to trace the age distribution, and in turn the star formation history in this region, located at the intersection of two spiral arms. A similar approach was also adopted by Efremov \& Elmegreen (1998, hereinafter EE98) and by Grebel \& Brandner (1998, hereinafter GB98), who also derived new PA relations on the basis of a larger sample of cluster Cepheids. By relying on these new relations, and on the large photometric database current available for LMC Cepheids -more than 1000 objects- they provided useful constraints on the star formation history of LMC over the last $200 \mathrm{Myr}$.

The main advantages in using the PA relation for estimating the Cepheid ages are the following: i) the age estimates rely upon an observable -the period- which is marginally affected by systematic errors such as reddening, distance, and photometric calibrations. ii) 
The PA relation can be applied to individual objects. Therefore, relative age estimates, based on this method, can supply accurate constraints on the occurrence of age gradients, since field Cepheids outnumber open clusters. iii) The application of the PA relation to cluster Cepheids supply the unique opportunity to estimate the age of the parent cluster, even though the photometry of main sequence turn-off stars is lacking or uncertain. However, empirical PA relations are affected by three drawbacks: i) current PA relations have been calibrated by using cluster Cepheids. However, both the zero-point and the slope of these relations are affected by uncertainties in the distance modulus and in the reddening of selected clusters, as well as by the period range covered by cluster Cepheids. ii) Cepheid PA relations have only been derived for fundamental Cepheids. The possibility to derive independent PA relations for fundamental and first overtone Cepheids would improve the accuracy of the PA relation in the short-period $(\log P \leq 1)$ range, because the intrinsic scatter is smaller. iii) Cepheid ages based on the PA relations rely on the statistical assumption that the Cepheid instability strip has a negligible temperature width. This is a plausible assumption in the short-period range, but the ages of long-period $(\log P>1)$ Cepheids should be estimated on the basis of a Period-Age-Color (PAC) relation. This relation accounts for individual Cepheid positions inside the instability strip, and therefore it is also marginally affected by the spread in luminosity due to evolutionary effects (M97). The reader interested in a detailed discussion concerning the width in temperature of the instability strip is referred to Bono et al. (2000a) and to Bono, Caputo, \& Marconi (2001).

The main aim of this investigation is to supply a new theoretical calibration of both PA and PAC relations for fundamental and first overtone Cepheids, by adopting homogeneous and detailed sets of nonlinear, convective pulsation models together with up-to-date evolutionary models. In order to constrain the accuracy of the new relations, we also plan to perform a detailed comparison between Cepheid pulsation ages and ages based on the fit of isochrones to turn-off stars of the parent cluster. This test will be performed on a sizable sample of Magellanic clusters hosting more than two Cepheids and two Galactic clusters.

In section 2, we describe the evolutionary and pulsational frameworks adopted in this investigation together with the procedures for computing current PA and PAC relations. In section 3, we present photometric data for cluster and Cepheid stars, and discuss the comparison between cluster and Cepheid ages. Finally, in section 4, we summarize our results and briefly outline future developments. 


\section{Theoretical models}

\subsection{Stellar evolutionary models}

The current evolutionary framework is based on the updated set of stellar evolutionary models recently computed by Pietrinferni et al. (2004). The reader interested in a detailed discussion concerning the input physics is referred to this paper. The main changes when compared with our previous investigation on intermediate-mass evolutionary models (Bono et al. 2000b) are the following:

- Opacity - Conductive opacity for electron degenerate matter was estimated following the prescriptions by Potekhin (1999).

- Energy loss rates - The energy loss rates for plasma-neutrino processes have been updated by using the most recent and accurate estimates provided by Haft, Raffelt \& Weiss (1994). For the other processes, we follow the prescriptions adopted by Cassisi \& Salaris (1997).

- Nuclear reaction rates - The nuclear reaction rates have been updated by using the NACRE database (Angulo et al. 1999), with the exception of the ${ }^{12} \mathrm{C}(\alpha, \gamma){ }^{16}$ O reaction. For this reaction we adopt the recent determination by Kunz et al. (2002). Electron screening is treated according to Graboske et al. (1973);

- EOS - The updated Equation of State (EOS) by A. Irwin ${ }^{1}$ has been used. It is noteworthy that this EOS allowed us to compute self-consistent stellar models in all evolutionary phases relevant for this investigation. For a thorough discussion concerning the key features of this EOS, see e.g. Cassisi, Salaris, \& Irwin (2003) and Irwin et al. (2004, in preparation).

- Convection - Superadiabatic convection is handled according to the Cox \& Giuli (1968) formalism of the mixing length theory (Böhm-Vitense 1958). The mixing length parameter has been fixed according to the solar calibration $(m l=1.713)$.

The efficiency of the physical mechanisms that govern the size of the convective core during central H-burning phases is still an open problem (see, e.g., Testa et al. 1999; Maeder \& Meynet 2000; Barmina, Girardi, \& Chiosi 2002; Brocato et al. 2003). Current stellar models do not account for core overshooting. The reader interested in

\footnotetext{
${ }^{1}$ The EOS code has been made available at ftp://astroftp.phys.uvic.ca under the GNU General Public License (GPL)
} 
a detailed discussion concerning the impact of mixing processes in intermediate-mass stars is referred to Castellani et al. (1985), Chiosi \& Maeder (1986), and Cassisi (2004).

- Chemical composition - The sets of evolutionary models have been computed for $\mathrm{Z}=0.004,0.008$, and $\mathrm{Z}=0.0198$ (solar). The initial He abundance was chosen according to Salaris et al. (2004) equal to $Y=0.245$. To reproduce the calibrated initial solar He-abundance we adopted $d Y / d Z \approx 1.4$ (Pietrinferni et al. 2004). The adopted He abundances for the three selected metal abundances are: $\mathrm{Y}=0.251,0.256$, and 0.273 .

- Stellar mass - The mass values of the evolutionary models adopted in this investigation range from $2.3 M_{\odot}$ to $10 M_{\odot}$. The mass loss is treated according to the Reimers formula (Reimers 1975) with $\eta$ equal to $0.4^{2}$.

Fig. 1 shows the H-R diagram for stellar models adopted in this investigation. Stellar isochrones have been computed with steps of 50 Myr for ages older than $200 \mathrm{Myr}$ and of 20 Myr for younger ages. Theoretical predictions have been transformed into the observational plane by adopting the bolometric corrections and the color-temperature transformations provided by Pietrinferni et al. (2004). The current set of stellar isochrones covers the typical age range of intermediate-mass stars with a very good resolution. Therefore, we also estimated the dependence of age on intrinsic parameters. In particular, we found:

$$
\log t=(8.911 \pm 0.018)+(0.423 \pm 0.002) \times M_{V}^{T O}+(0.200 \pm 0.008) \times \log Z \quad r^{2}=0.99 \quad[1]
$$

where $\mathrm{t}$ is the age in $\mathrm{yr}, M_{V}$ the TO visual magnitude, $r$ the correlation parameter, and the other symbols have their usual meaning. Note that current analytical relation applies to stellar structures with ages ranging from $30 \mathrm{Myr}$ to $1 \mathrm{Gyr}$, and for the selected chemical compositions (see Table 5).

\subsection{Pulsation models}

We adopted the sequences of nonlinear, convective pulsation models constructed by Bono et al. (1999, 2000a,2002a) to estimate the PA and the PAC relations. This set of models was computed by adopting chemical compositions typical of Galactic $(Y=0.28, Z=0.02)$ and Magellanic Cepheids $(\mathrm{Y}=0.25, \mathrm{Z}=0.004$, Small Magellanic Cloud [SMC]; $\mathrm{Y}=0.25, \mathrm{Z}=0.008$,

\footnotetext{
${ }^{2}$ The entire evolutionary database can be found at the following URL address: http://www.te.astro.it/BASTI/index.php.
} 
Large Magellanic Cloud [LMC]) and cover a wide range of stellar masses and effective temperatures. Note that the He abundances adopted to construct the pulsation models present a mild difference when compared with the evolutionary ones (see $\$ 2.1$ ). However, such a difference has a marginal impact upon pulsation properties, and in turn on the conclusions of our current investigation. The input parameters adopted for fundamental and first overtone models are listed in Table 1. Both canonical and noncanonical Cepheid models were constructed by adopting the same mass-luminosity $(\mathrm{M} / \mathrm{L})$ relations used by Bono et al. (1999). The grid of canonical Cepheid models was implemented with new sequences for $M / M_{\odot}=9.4, \log L / L_{\odot}=4.4, Z=0.004$ and for $M / M_{\odot}=10.1, \log L / L_{\odot}=4.4$ and $Z=0.008$. On the basis of these models, we performed a linear least-squares fit to luminosities and effective temperatures, i.e. $\log T_{e}=\alpha+\beta \log L / L_{\odot}$, of blue and red edges of both fundamental and first overtone instability strip. The analytical relations for the three different chemical compositions are listed in Table 2. Note that the periods of fundamental pulsators range from $\log P=0.17$ to $\log P=2.02$, while for first overtones they range from $\log P=-0.20$ to $\log P=0.88$. This Table also gives the analytical relations for the effective temperature of the fundamental instability strip at solar chemical composition provided by Petroni et al. (2003). These models have been constructed by adopting the same treatment for turbulent convection, but with different assumptions concerning input physics and spatial resolution across the ionization regions. For these models a M/L relation based on more recent canonical evolutionary models was also adopted. A glance at the two different sets of predictions (see Table 2) indicates that the difference is at most of the order of 0.02 dex in the zero-point and smaller than 0.01 dex in the slope. This finding suggests that the temperature of the edges marginally depends on these parameters. However, recent empirical evidence (Tammann, Sandage, \& Reindl 2003; Kanbur \& Ngeow 2004; Sandage, Tammann, \& Reindl 2004) suggests that the PL relations for fundamental Cepehids, and in turn, the edges of the Cepheid instability strip show a change in the slope at $\log P \approx 1$. According to theoretical predictions based on convective models, this occurrence is caused by a twofold effect. In the long-period range $(\log P>1.5)$ the instability strip moves toward redder colors causing a flattening in the PL relation (Bono \& Marconi 1998; Bono et al. 2000a). This behavior is supported by observations (Sasselov et al. 1997). In the short-period range $(\log P<0.4)$, the slope of the fundamental red edge becomes shallower, and in turn, the width in temperature of the instability strip becomes narrower (see Fig. 2 in Bono et al. 2001). This trend is also supported by observations (Bauer et al. 1999; Udalski et al. 1999a,b). To account for these effects, we also computed quadratic analytical relations connecting the effective temperature of the instability edges to the stellar luminosity (see Table 3). The quadratic fit has only been performed for canonical models, since for this set we computed a finer grid of models. Note that the difference between linear and quadratic relations is at most 0.05 dex, and therefore the impact on the PA and the PAC relations is negligible. Obviously, the 
difference increases as soon as we move from the theoretical to the observational plane, due to changes in the bolometric correction. We did not estimate quadratic relations for first overtones, because both theory and observations suggest that the instability edges for these pulsators are linear. The difference between fundamental and first overtone Cepheids is due to the narrower period and color range covered by the latter pulsators.

The current analytical relations and evolutionary tracks (see §2.1) were adopted to select the evolutionary phases falling inside the instability strip. This means that we selected not only the second and the third crossing, but also the first one. Finally, the PA relations were estimated as a linear fit by weighing each individual evolutionary phase for the amount of time spent inside the instability strip. The coefficients of the PA relations as a function of the chemical composition are listed in Table 4. Fig. 2 shows the new PA relations for fundamental (bottom) and first overtone (top) Cepheid models. Data plotted in the bottom panel disclose that the dependence on the metal content is strictly decreasing when moving from short (old) to long-period (young) Cepheids. On the other hand, data for first overtone pulsators (top panel) display a different behavior for periods longer than $\log P \geq 0.5$. This change is due to the narrowing in temperature of the first overtone instability strip when moving toward higher luminosities (Bono et al. 2002b). This prediction agrees quite well with observations, and indeed the period distribution of the first overtone pulsators in the Magellanic Clouds shows a well-defined cut-off toward longer periods (Udalski et al. 1999a,b; Beaulieu \& Marquette 2000). This region of the instability strip is a robust observable, since it can be adopted to constrain the luminosity above which Cepheids only pulsate in the fundamental mode (Bono et al. 2002b). Moreover, the metallicity effect among the different first overtone PA relations appears negligible, within the intrinsic dispersions.

Fig. 3 shows the comparison between current predictions for solar chemical composition and similar relations available in the literature. The PA relations plotted in this figure show a reasonable agreement, in particular if we account for the fact that they have been derived using different approaches to calibrate the absolute zero-point, as well as different sets of evolutionary models. As expected, our PA relation supplies, at fixed period, younger ages because the evolutionary tracks we adopted do not account for convective overshooting. To estimate on a quantitative basis the age difference between evolutionary tracks which either account for or neglect convective core overshooting, the current set of stellar models has been implemented with an additional set of evolutionary tracks with stellar masses ranging from 4 to $10 M_{\odot}$. These tracks were constructed by adopting a significant amount 
of core overshooting $\left(\lambda_{c}=0.2 H_{p}{ }^{3}\right)$ and $\lambda_{e}$ (envelope overshooting ${ }^{4}$ ) ranging from 0 to $0.5 H_{p}$ during hydrogen and helium burning phases (Pietrinferni et al. 2004). For each given mass value and chemical composition, we estimated a mean luminosity and a mean temperature typical of the instability strip, i.e. $3.81 \leq \log T_{e} \leq 3.85$, according to the shape of the blue loop. On the basis of the predicted stellar mass, luminosity, and effective temperature, we estimated a mean fundamental period for both canonical and noncanonical models using the pulsation relations provided by Bono et al. (2000a). Data plotted in Fig. 4 (see also Table 5) indicate that the difference in age for the selected stellar masses ranges from $7 \%$ $\left(8 M_{\odot}, \mathrm{Z}=0.008, \mathrm{Y}=0.25\right)$ to $18 \%\left(10 M_{\odot}, \mathrm{Z}=0.008, \mathrm{Y}=0.25\right)$. Note that for $M=4 M_{\odot}$ $\mathrm{Z}=0.004$ and $M=5 M_{\odot}, \mathrm{Z}=0.02$ the noncanonical evolutionary tracks do not perform the loop. Interestingly enough, these are the typical differences between current PA relations and those based on evolutionary models which account for convective core overshooting (M97; GB98).

To further improve the accuracy of Cepheid age estimates, in particular in the longperiod range, we computed new analytical relations accounting for the width in temperature of the instability strip. The coefficients of the PAC relations are listed in Table 6 as a function of the chemical composition. Note that current PAC relations have been computed, for each given chemical composition, for optical colors, namely B-V and V-I. Fig. 5 shows the projection onto a plane of the new theoretical PAC relations. To estimate the difference between Cepheid ages based on the PA and on the PAC relation we computed the age difference for each given composition along two isoperiodic lines at $\log P=1$ and $\log P=1.5$. Table 7 gives for the two selected periods the age based on the PA relation (columns 2,5,8), and the age based on the PAC relation along the blue (columns 3,6,9) and the red (columns $4,7,10$ ) edge of the instability strip. Table 7 also gives the logarithmic temperatures (number in parentheses) of the instability edges in which the period is equal to $\log P=1$ or 1.5 . Data listed in this table indicate that the age difference ranges from $\Delta \log t=0.04-0.07$ for hotter Cepheids to $\Delta \log t=-0.01--0.04$ for cooler ones.

\footnotetext{
${ }^{3}$ The extent of the non-canonical mixed region is usually defined in terms of a parameter, $\lambda$, which fixes the length, in units of the local pressure scale height $H_{p}$, covered by convective cells into the stable region surrounding the convective core.

${ }^{4}$ It is worth noting that envelope overshooting at odds with core overshooting, has no effect on the evolutionary lifetimes, but it substantially affects the morphology of the blue loops during the core Heburning phases.
} 


\section{Empirical data}

In order to validate current PA and PAC relations, we decided to perform a detailed comparison between age estimates based on the canonical Main-Sequence fitting, and on periods and colors of cluster Cepheids. Photometric data collected by OGLE for Magellanic cluster Cepheids (Udalski et al. 1999a,b; Pietrzynski \& Udalski 1999) are very useful to accomplish this goal. These data are very homogeneous, since they have been collected with the same observational equipment, and reduced by adopting the same approach. Moreover, and even more importantly, static and variable stars have the same absolute photometric zero-point. To perform the test we selected LMC and SMC clusters hosting at least two cluster Cepheids. We ended up with a sample of 15 and 12 clusters respectively.

Figures 6a,d and 7a, $\mathrm{c}^{5}$ show the fit of stellar isochrones at fixed metal-content with cluster stars in two different Color-Magnitude Diagrams, namely V,B-V and V,V-I. Circles and squares mark fundamental and first overtone cluster Cepheids, according to the classification and the membership provided by Pietrzynski \& Udalski (1999). Individual cluster age estimates have been performed by assuming the same distance modulus (18.5 for LMC clusters and 19.00 for SMC clusters), and the mean reddening estimate based on cluster Cepheids provided by Pietrzynski \& Udalski (1999). It is worth mentioning that we did not force stellar isochrones to fit both magnitude and colors of classical Cepheids. The extent in color of the so-called blue loop depends on several physical parameters and assumptions adopted to construct intermediate-mass evolutionary models (Chiosi, Bertelli, \& Bressan 1992; Stothers \& Chin 1993,1994; Bono et al. 2000b). As a whole, the agreement between the fit in the $\mathrm{V}, \mathrm{B}-\mathrm{V}$ and in the $\mathrm{V}, \mathrm{V}$-I planes is quite good. The color excess in V-I was estimated from $\mathrm{E}(\mathrm{B}-\mathrm{V})$ according to Cardelli et al. (1988). Table 8 lists the new cluster age estimates, together with the mean reddening value. To provide a plausible estimate of the formal error on isochrone ages, we assumed an uncertainty of 0.15 mag on the distance that accounts for uncertainty on the individual cluster distances and on reddening estimates and of 0.2 dex on the cluster metallicity. By accounting for these uncertainties and the intrinsic errors on the coefficients of equation 1, we end up with a tipical uncertainty on the cluster age of the order of 0.075 dex.

At the same time, we estimated the cluster ages using both the PA and the PAC relations for fundamental and first overtone Cepheids. Individual cluster ages are listed in Table 8, together with the formal uncertainties given by the uncertainties on the coefficients of PA and PAC relations. Figures 8 and 9 display the relative age difference between evolutionary (isochrones) and pulsation ages for LMC and SMC clusters, respectively. Interestingly

\footnotetext{
${ }^{5}$ The figures $6 \mathrm{~b}, \mathrm{c}, \mathrm{d}$ and $7 \mathrm{~b}, \mathrm{c}$ are only available in the on-line edition of the manuscript.
} 
enough, data plotted in these figures show that, within current empirical and theoretical uncertainties (see error bars), evolutionary and pulsation cluster ages agree on average at the level of $20 \%$ for LMC clusters and $10 \%$ for SMC clusters. The difference between the two sets, and in particular the mild increase in the LMC Cepheid ages based on the PAC relation (top panel Fig. 8), might be due to uncertainties in the reddening correction (Zaritsky et al. 2002, and references therein) and/or in the cluster membership, since LMC clusters are typically located across the bar.

In order to provide a comprehensive analysis over a broad metallicity range, we also investigated two Galactic clusters hosting at least two classical Cepheids, namely NGC 6067 and NGC 7790. By adopting periods, mean magnitudes, and individual reddenings provided by Berdnikov (2000) for Cepheids in NGC 7790 (CEa Cas, CEb Cas, CF Cas) and by Laney \& Stobie (1994) for Cepheids in NGC 6067 (QZ Nor, V430 Nor) we found that mean cluster ages are $\log t=7.85 \pm 0.02 \mathrm{yr}(\mathrm{PA})$ and $7.81 \pm 0.02 \mathrm{yr}(\mathrm{PAC})$ for NGC 7790, as well as $\log t=7.76 \pm 0.22 \mathrm{yr}(\mathrm{PA})$ and $7.79 \pm 0.11 \mathrm{yr}(\mathrm{PAC})$ for NGC 6067 (see Table 9 ). It is worth noting that Romeo et al. (1989), by using a period-age relation based on evolutionary tracks accounting for convective core overshooting and mass loss, estimated an age of $\log t=8.1$ yr for Cepheids in NGC 7790.

As far as the cluster photometry is concerned, we adopted the B,V,I data available in the web site developed and maintained by J.-C. Mermilliod for stellar systems

(http://obswww.unige.ch/webda/webda.html). Original B,V data for NGC 6067 have been collected by Walker \& Coulson (1985), while V,I data have been collected by Piatti, Claria, \& Bica (1998). At the same time, B,V,I data NGC 7790, have been collected by Phelps, \& Janes (1994), Gupta et al. (2000), and Henden (2003,

ftp://ftp.nofs.navy.mil/pub/outgoing/aah/sequence/). Fig. 10 shows the fit between empirical data and stellar isochrones at solar chemical composition. The cluster distances adopted to perform the fit were estimated using the recent calibration of the optical PL relations for Galactic Cepheids, recently provided by Storm et al (2004), and the apparent magnitudes and reddenings listed in Table 9. We found that the true distance moduli are $11.27 \pm 0.35$ for NGC 6067 and 12.50 \pm 0.23 for NGC 7790. By adopting these distances and mean cluster reddenings of 0.32 (NGC 6067, Coulson \& Caldwell 1985) and 0.53 (NGC 7790, Lee \& Lee 1999), we estimated cluster ages of $70 \pm 10$ and $80 \pm 10 \mathrm{Myr}$, respectively. Once again, the difference between pulsational and evolutionary ages is smaller than $20 \%$. 


\section{Summary and final remarks}

We computed new PA and PAC relations for fundamental and first overtone Cepheids for chemical compositions typical of Magellanic and Galactic Cepheids. The current predictions rely on homogeneous sets of stellar isochrones and pulsation models covering the entire range of intermediate-mass stars. We found that the metallicity has a marginal effect on the PA relation. To validate current theoretical scenario, we performed a detailed comparison between evolutionary (isochrone fit) and pulsation (PA and PAC relations) ages of a sizable sample of LMC and SMC clusters hosting at least two Cepheids. In order to avoid deceptive uncertainties in the photometric calibration of both Cepheids and cluster stars, we adopted the homogeneous B,V,I photometric catalog provided by the OGLE collaboration (Pietrzynski \& Udalski 1999). The isochrone fit was performed both in the V,B-V and in the V,V-I CMDs by adopting the reddening estimated by Pietrzynski \& Udalski (1999) for cluster Cepheids. We found that pulsational and evolutionary cluster ages agree quite well, and indeed the difference in age ranges from $10 \%$ to $20 \%$. We performed the same comparison for two Galactic clusters, namely NGC6067 and NGC7079. We found once again that the difference between pulsation and evolutionary ages is smaller than $20 \%$.

These findings support the evidence that PA and PAC relations supply accurate individual age estimates. This means that they can be adopted to trace the age distribution not only in the Galactic disk, but also across the main body of the Magellanic Clouds. It is worth mentioning that the use of independent relations for fundamental and first overtone Cepheids will supply more accurate age estimates in the short-period $(\log P<1)$ range, since the intrinsic dispersion is smaller. At the same time, the use of the PAC relations will supply more accurate age estimates in the $\operatorname{long}$-period $(\log P>1)$ range. Note that the vertical error bars in Fig. 8 and 9 mainly depend on the number of cluster Cepheids. Individual Cepheid ages based on the PAC relation are more accurate than the ages based on the PA relation, as soon as the uncertainty on the reddening correction is on average smaller than 0.08 mag for B-V colors and 0.07 mag for V-I colors. These limits have been estimated by accounting for the color coefficients listed in Table 6, and the age differences between PA and PAC relations listed in Table 7. The theoretical framework we developed can be adopted to detect the occurrence of age gradient(s), not only across star-forming regions, but also along the spiral arms of external galaxies. The sizable sample of extragalactic Cepheids detected by the two HST Key Projects aimed at estimating the Hubble constant (Freedman et al. 2001; Saha et al. 2001) appears a well-suited extension. The same applies to the large sample of Cepheids discovered in M31 and M33 by the DIRECT experiment (Bonanos et al. 2003, and references therein).

Finally, we would also like to mention that well-observed open clusters hosting sizable 
Cepheid samples can be safely adopted to test the accuracy of the physical ingredients currently adopted to construct evolutionary models. The difference between the cluster age based on the luminosity function and the pulsation ages based on PA and PAC relations can supply tight constraints on He-burning phases. Note that these two methods do not depend at all upon the cluster distance.

It is a pleasure to thank V. Castellani for several thorough discussions and a detailed reading of an early draft of this manuscript. We are also very grateful to A. Bragaglia for many useful suggestions concerning the photometric data of Galactic open clusters. We wish also to thank an anonymous referee for his/her detailed suggestions and helpful comments which improved both the content and the readability of this paper. We acknowledge J.-C. Mermillod and A. Henden for making available the photometric data bases they collected. This work was partially supported by MIUR/COFIN 2002 under the project (\#028935): "Stellar Populations in Local Group Galaxies" and by INAF under the project: "The Large Magellanic Cloud". WG and GP gratefully acknowledge financial support for this work from the Chilean Center for Astrophysics FONDAP 15010003. 


\section{REFERENCES}

Alexander, D. R.; Ferguson, J. W. 1994, ApJ, 437, 879

Angulo, C. et al. 1999, NuPh, 656, 3

Barmina, R., Girardi, L., \& Chiosi, C. 2002, A\&A, 385, 847

Bauer, F., et al. 1999, A\&A, 348, 175

Beaulieu, J.-P.; Marquette, J.-B. 2000, in IAU Colloq. 176, The Impact of Large-Scale Surveys on Pulsating Star Research, ed. L. Szabados \& D. Kurtz (San Francisco: ASP), 139

Böhm-Vitense, E. 1958, ZA, 46, 108

Bonanos, A. Z., Stanek, K. Z., Sasselov, D. D., Mochejska, B. J., Macri, L. M., Kaluzny, J. 2003, AJ, 126, 175

Bono, G., Caputo, F., Cassisi, S., Marconi, M., Piersanti, L., \& Tornambè, A. 2000b, ApJ, 543,955

Bono, G., Caputo, F., \& Marconi, M. 2001, MNRAS, 325, 1353

Bono, G., Castellani, V., \& Marconi, M. 2000a, ApJ, 529, 293

Bono, G., Castellani, V., \& Marconi, M. 2002a, ApJ, 565, L83

Bono, G., Groenewegen, M. A. T., Marconi, M., \& Caputo, F. 2002b, ApJ, 574, L33

Bono, G., Marconi, M. 1998, in A Half Century of Stellar Pulsation Interpretation: A Tribute to A.N. Cox, ed. P.A. Bradley \& Joyce A. Guzik, (San Francisco: ASP), 287

Bono, G., Marconi, M., \& Stellingwerf, R. F. 1999, ApJS, 122, 167

Brocato, E., Castellani, V., Di Carlo, E., Raimondo, G., \& \& Walker, A. R. 2003, AJ, 125, 3111

Cardelli, J. A., Clayton, G. C., \& Mathis, J. S. 1988, ApJ, 329, L33

Cassisi, S. 2004, in IAU Colloq. 193, Variable Stars in the Local Group, ed. D.W. Kurtz \& K.R. Pollard (San Francisco: ASP), 489

Cassisi, S., \& Salaris, M. 1997, MNRAS, 285, 593 
Cassisi, S.; Salaris, M.; Irwin, A. W. 2003, ApJ, 588, 862

Castellani, V., Chieffi, A., Tornambé, A., \& Pulone, L. 1985, ApJ, 294, L31

Chiosi, C., Bertelli, G., \& Bressan, A. 1992; ARA\&A, 30, 235

Chiosi, C., \& Maeder, A. 1986, ARA\&A, 24, 329

Coulson, I. M. \& Caldwell, J. A. R. 1985, MNRAS, 216, 671

Cox, J. P., \& Giuli, R. T. 1968, in Principles of stellar structure (Gordon and Breach: New York), 262

Efremov, Y. N. 1978, SvA, 22, 161

Efremov, Y. N., \& Elmegreen, B. G. 1998, MNRAS, 299, 588 (EE98)

Elmegreen, B. G., \& Efremov, Y. N. 1996, ApJ, 466, 802

Feast, M. 1999, PASP, 111, 775

Freedman, W. L., HST Key Project 2001, ApJ, 553, 47

Fouqué, P., Storm, J., Gieren, W. 2003, in Stellar Candles for the Extragalactic Distance Scale, ed. D. Alloin \& W. Gieren (Springer-Verlag: Berlin), 21

Graboske, H. C., Dewitt, H. E., Grossman, A. S., Cooper, M. S. 1973, ApJ, 181, 457

Grebel, E. K.; Brandner, W. 1998, in The Magellanic Clouds and Other Dwarf Galaxies, eds. T. Richtler \& J.M. Braun, (Shaker-Verlag: Aachen), 151 (GB98)

Grevesse, N., \& Noels, A. 1993, in Origin and Evolution of the Elements, ed. N. Prantzos, E. Vangioni-Flam, \& M. Cassè (Cambridge: Cambridge Univ. Press), 14

Gupta, A.C., Subramaniam, A., Sagar, R., Griffiths, W.K. 2000, A\&AS, 145, 365

Haft, M., Raffelt, G., \& Weiss, A. 1994, ApJ, 425, 222

Iglesias, C. A., \& Rogers, F. J. 1996, ApJ, 464, 943

Kanbur, S.M., \& Ngeow, C.-C. 2004, MNRAS, 350, 962

Kippenhahn, R. \& Smith, L. 1969, A\&A, 1, 142

Kraft, R. P., \& Schmidt, M. 1963, ApJ, 137, 249 
Kunz, R. et al. 2002, ApJ, 567, 643

Lee, J. \& Lee, S. 1999, JKAS, 32, 91

Magnier, E. A., Prins, S., Augusteijn, T., van Paradijs, J., Lewin, W. H. G. 1997, A\&A, 326, 442 (M97)

Maeder, A., \& Meynet, G. 2000, ARA\&A, 38, 143

Meyer-Hofmeister, E. 1969, A\&A, 2, 143

Petroni, S.; Bono, G.; Marconi, M.; Stellingwerf, R. F. 2003, ApJ, 599, 522

Phelps, R. L., \& Janes, K. A. 1994, ApJS, 90, 31

Pietrinferni, A., Cassisi, S., Salaris, M., \& Castelli, F. et al. 2004, ApJ, 612, 168

Piatti, A. E., Claria, J. J., Bica, E. 1998, ApJS, 116, 263

Pietrzynski, G., \& Udalski, A. 1999, AcA, 49, 543

Potekhin, A. Y. 1999, A\&A, 351, 787

Reimers, D. 1975, in Problems in stellar atmospheres and envelopes, (Springer-Verlag: Berlin), 229

Romeo, G., Bonifazi, A., Fusi Pecci, F., \& Tosi, M. 1989, MNRAS, 240, 459

Saha, A., Sandage, A., Tammann, G. A., Dolphin, A. E., Christensen, J., Panagia, N., Macchetto, F. D. 2001, ApJ, 562, 314

Salaris, M., Riello, M., Cassisi, S., \& Piotto, G. 2004, A\&A 422, 217

Sandage, A., Tammann, G. A., \& Reindl, B. 2004, A\&A, 424, 43

Sasselov, D. D. et al. 1997, A\&A, 324, 471

Storm, J., Carney, B. W., Gieren, W. P., Fouqué, P., Latham, D. W., Fry, A. M. 2004, A\&A, 415,531

Stothers, R. B., \& Chin, C. W. 1993, ApJ, 412, 294

Stothers, R. B., \& Chin, C. W. 1994, ApJ, 431, 797 ‘

Tammann, G. A., Sandage, A., \& Reindl, B. 2003, A\&A, 404, 423 
Testa, V., Ferraro, F. R., Chieffi, A., Straniero, O., Limongi, M., Fusi Pecci, F. 1999, AJ, 118,2839

Tsvetkov, Ts. G. 1989, Ap\&SS, 151, 39

Udalski, A., Soszynski, I., Szymanski, M., Kubiak, M., Pietrzynski, G., Wozniak, P., \& Zebrun, K. 1999a, AcA, 49, 223

Udalski, A., Soszynski, I., Szymanski, M., Kubiak, M., Pietrzynski, G., Wozniak, P., \& Zebrun, K. 1999b, AcA, 49, 437

Udalski, A., Szymanski, M., Kubiak, M., Pietrzynski, G., Soszynski, I., Wozniak, P., Zebrun, K. 1999c, AcA, 49, 201

Walker, A. R., Coulson, I. M. 1985, South African Astron. Obs. Circ. 9, 97

Zaritsky, D., Harris, J., Thompson, I. B., Grebel, E. K., \& Massey, P. 2002, AJ, 123, 855 


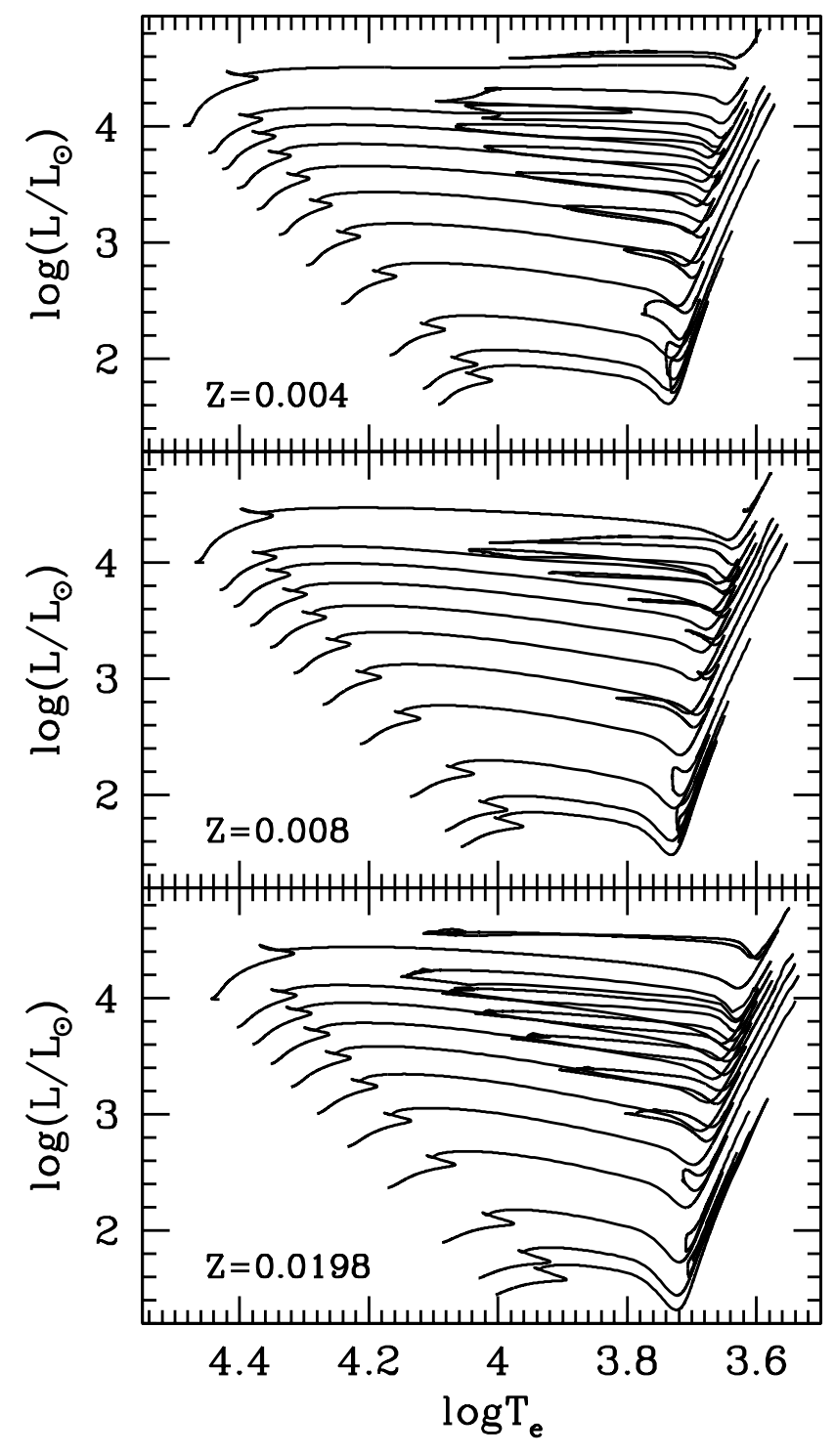

Fig. 1.- Theoretical H-R diagram for intermediate-mass stars with different chemical compositions (see labeled values). See text for more details. 

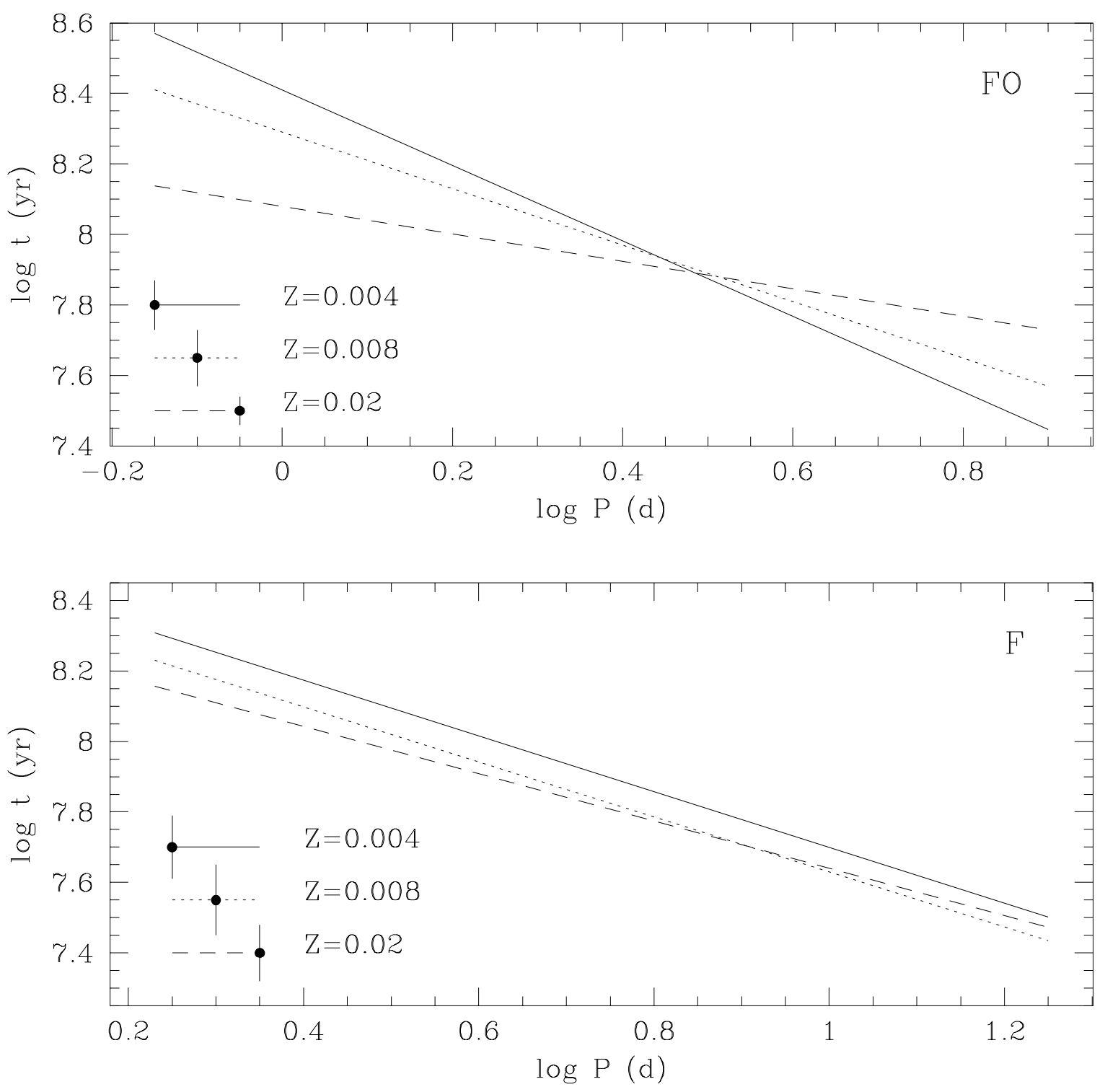

Fig. 2.- Predicted Period-Age relations for first-overtone (top) and fundamental (bottom) Cepheids with different chemical compositions (see labeled values). The vertical bars display the standard deviation of analytical relations. 


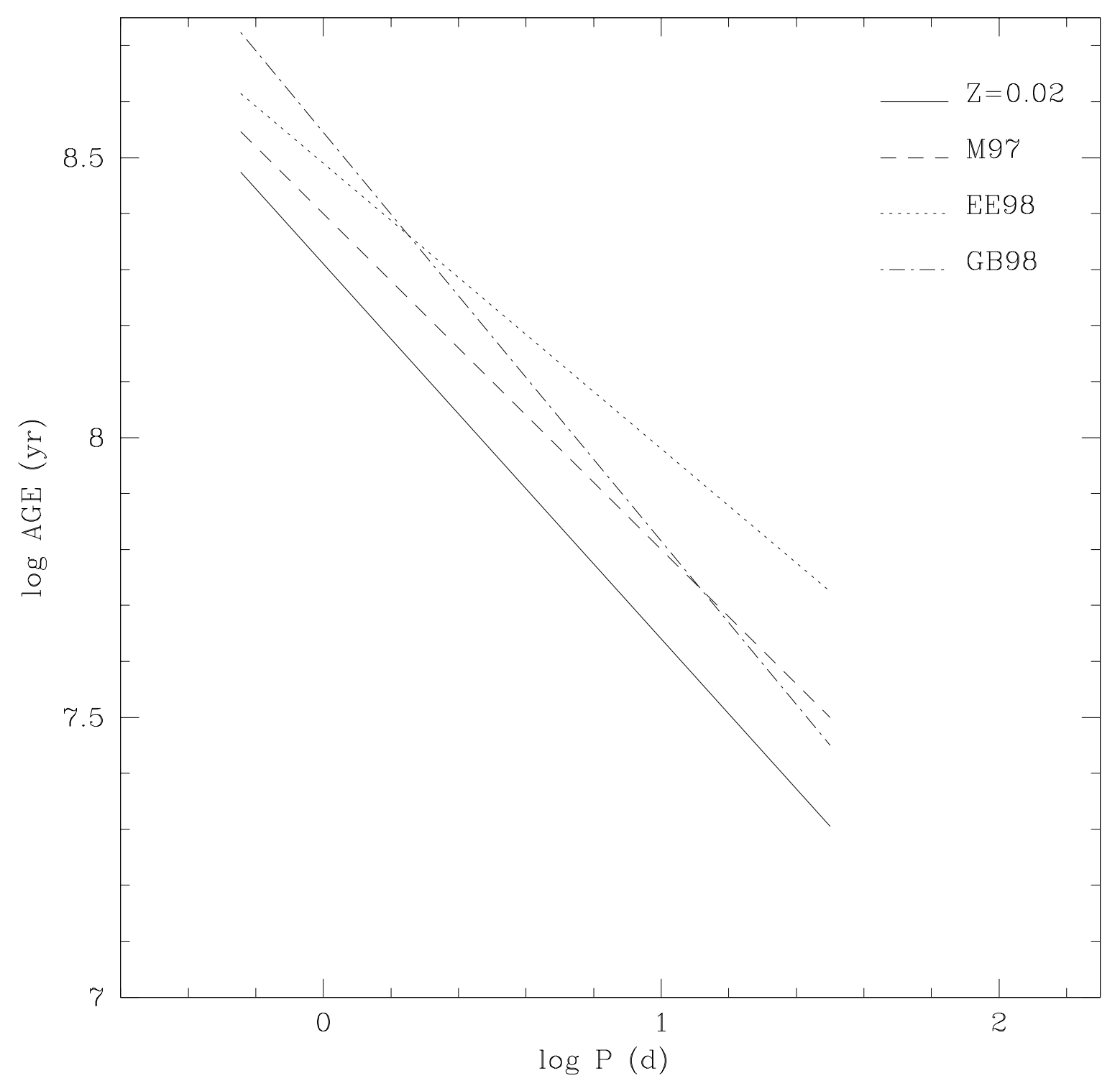

Fig. 3.- Comparison between current Period-Age relation at solar chemical composition (solid line) and similar relations available in the literature. The dashed line shows the PA relation provided by Magnier et al. (1997), while the dotted and the dashed-dotted lines the PA relations by Efremov \& Elmegreen (1998) and by Grebel \& Brandner (1998), respectively. Note that PA relations plotted in this figure have been obtained using different evolutionary models. See text for more details. 


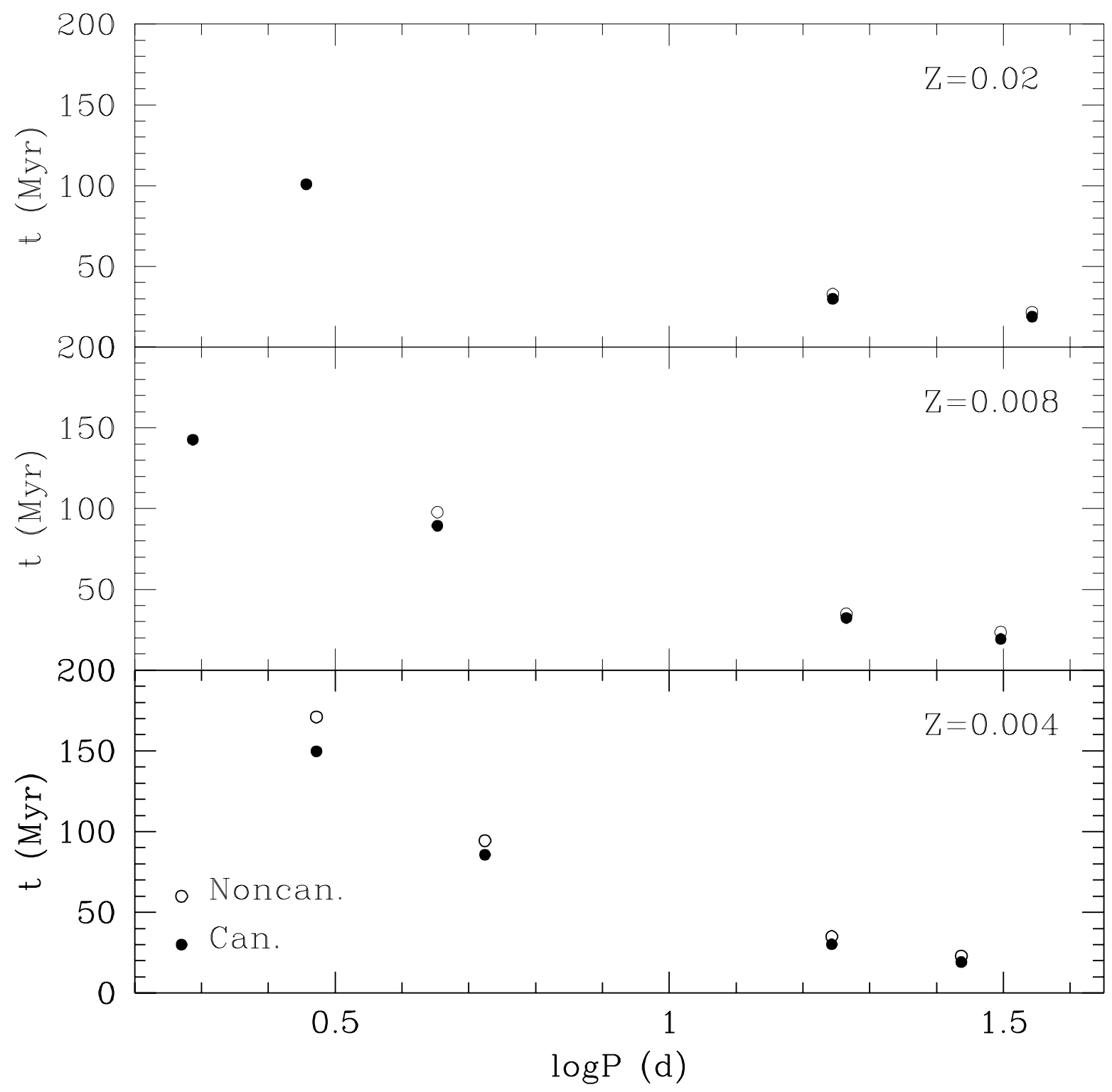

Fig. 4.- Evolutionary ages (Myr) as a function of pulsation period (days) for different chemical compositions (see labeled values). Circles refer to fundamental periods estimated according to evolutionary predictions that either neglect (Canonical, filled) or account (Noncanonical, open) for convective core overshooting. 


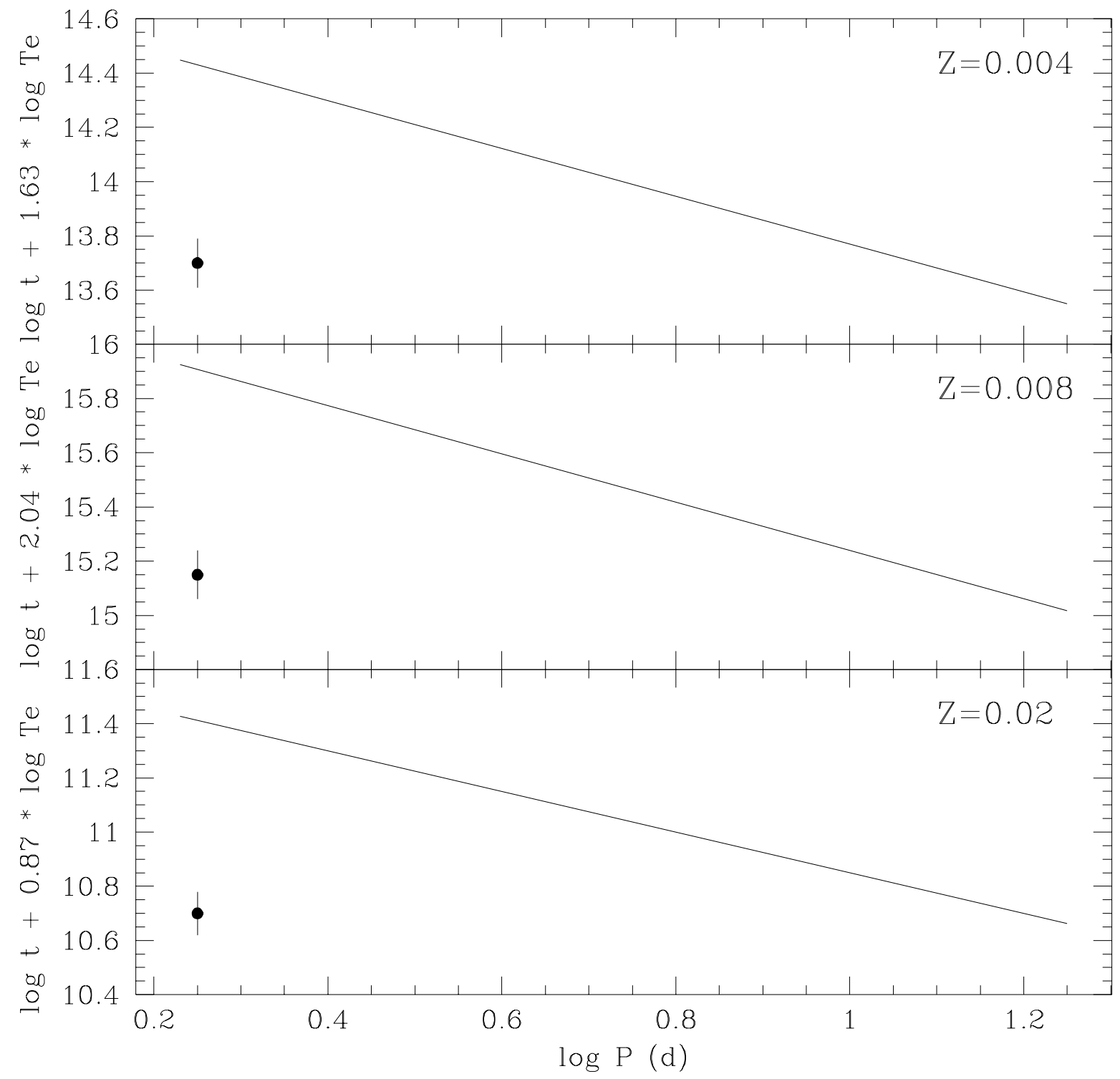

Fig. 5.- Projection onto a plane of Period-Age-Color relations for fundamental Cepheids at different chemical compositions (see labeled values). 


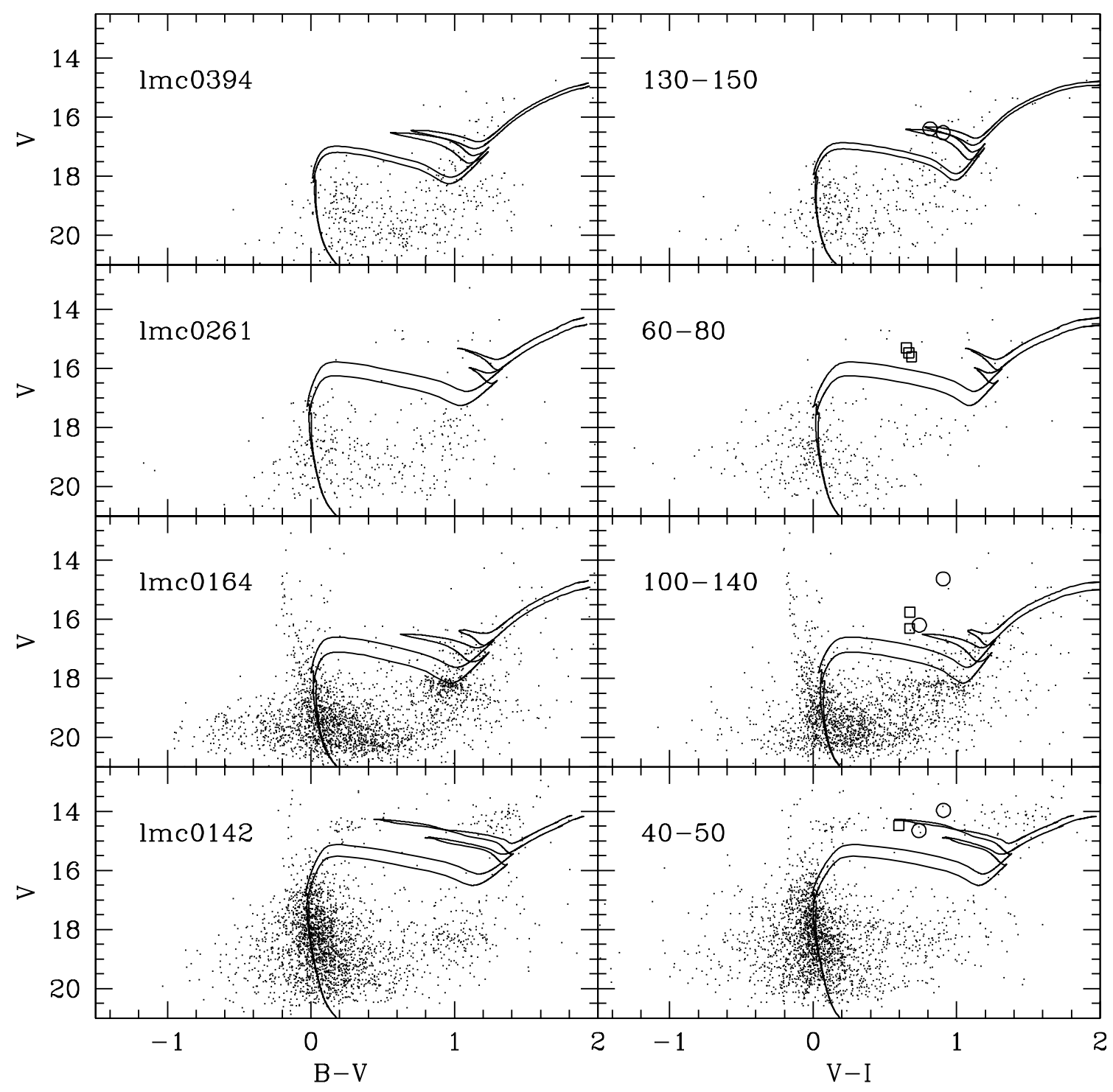

Fig. 6a.- Fit of empirical Color-Magnitude Diagrams in V,B-V (left) and in V,V-I (right) of LMC clusters with stellar isochrones at fixed metal content $(\mathrm{Z}=0.01)$. Open circles and squares mark fundamental and first overtone cluster Cepheids respectively. Individual cluster ages, and reddening values are listed in Table 6 . 


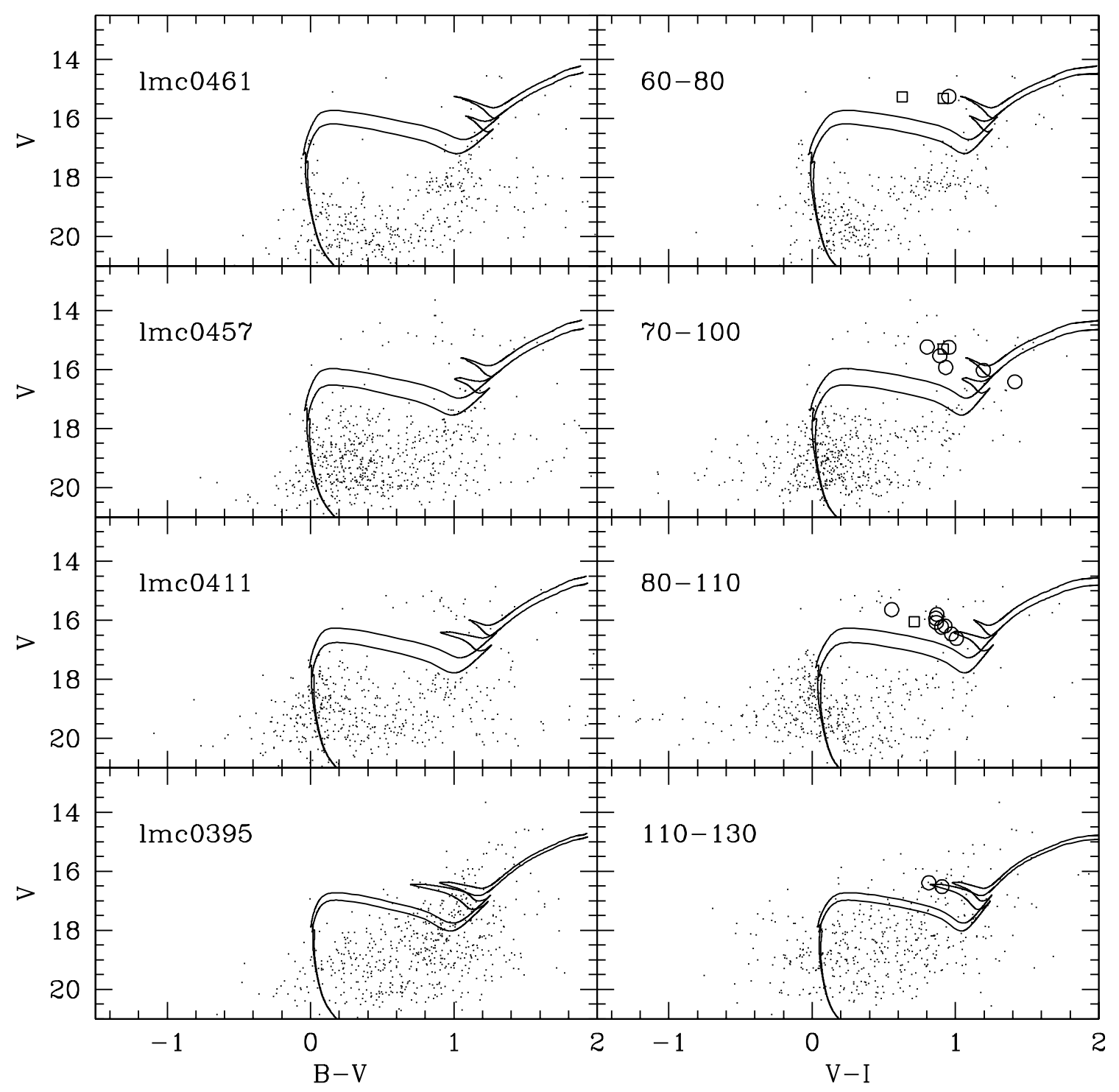

Fig. 6b.- Same as Fig. 6a but for a different sample of LMC clusters. 


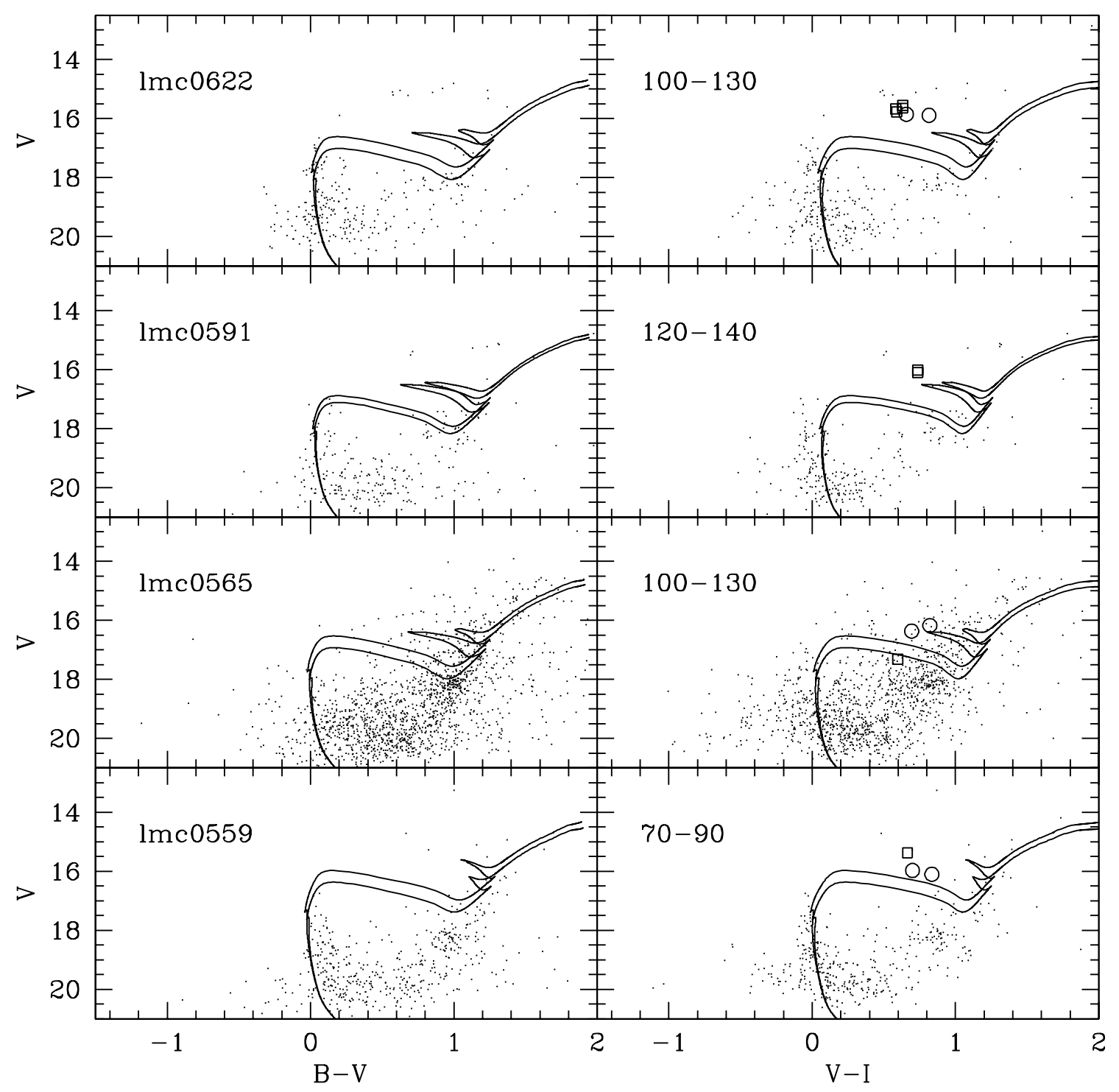

Fig. 6c.- Same as Fig. 6a but for a different sample of LMC clusters. 


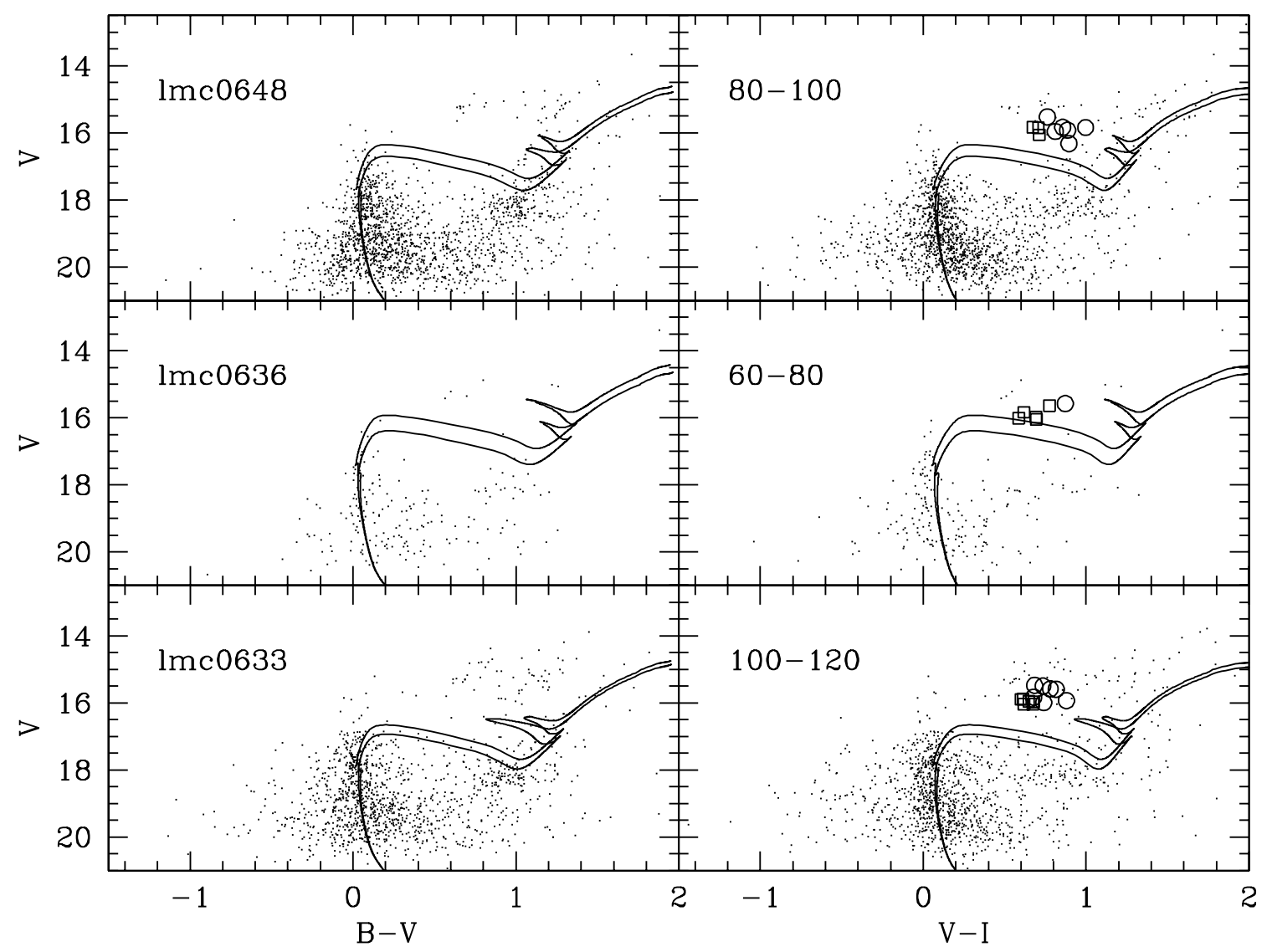

Fig. 6d.- Same as Fig. 6a but for a different sample of LMC clusters. 


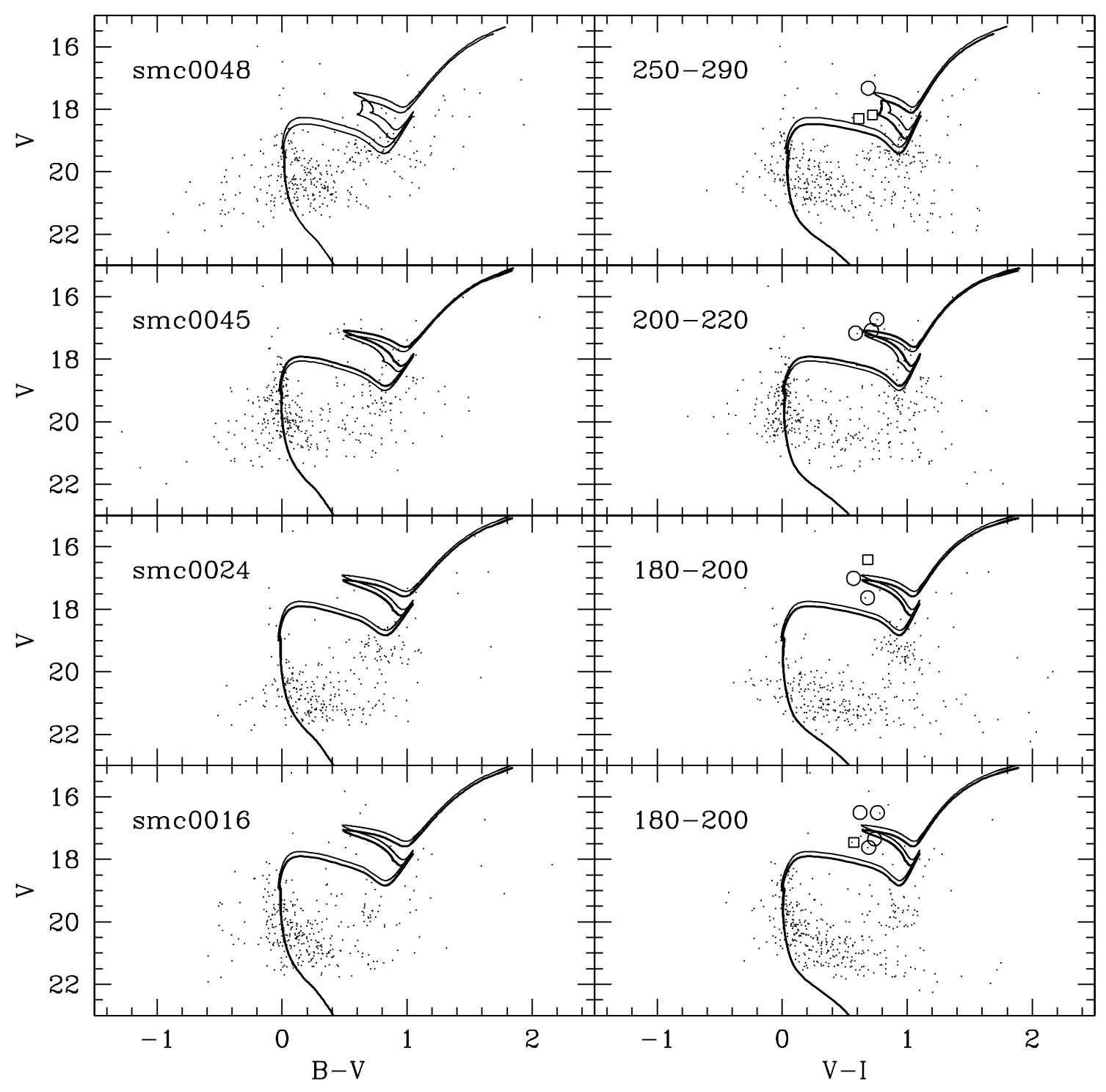

Fig. 7a.- Same as Fig. 6a but for a sample of SMC clusters and stellar isochrones with a metal content of $\mathrm{Z}=0.004$. 


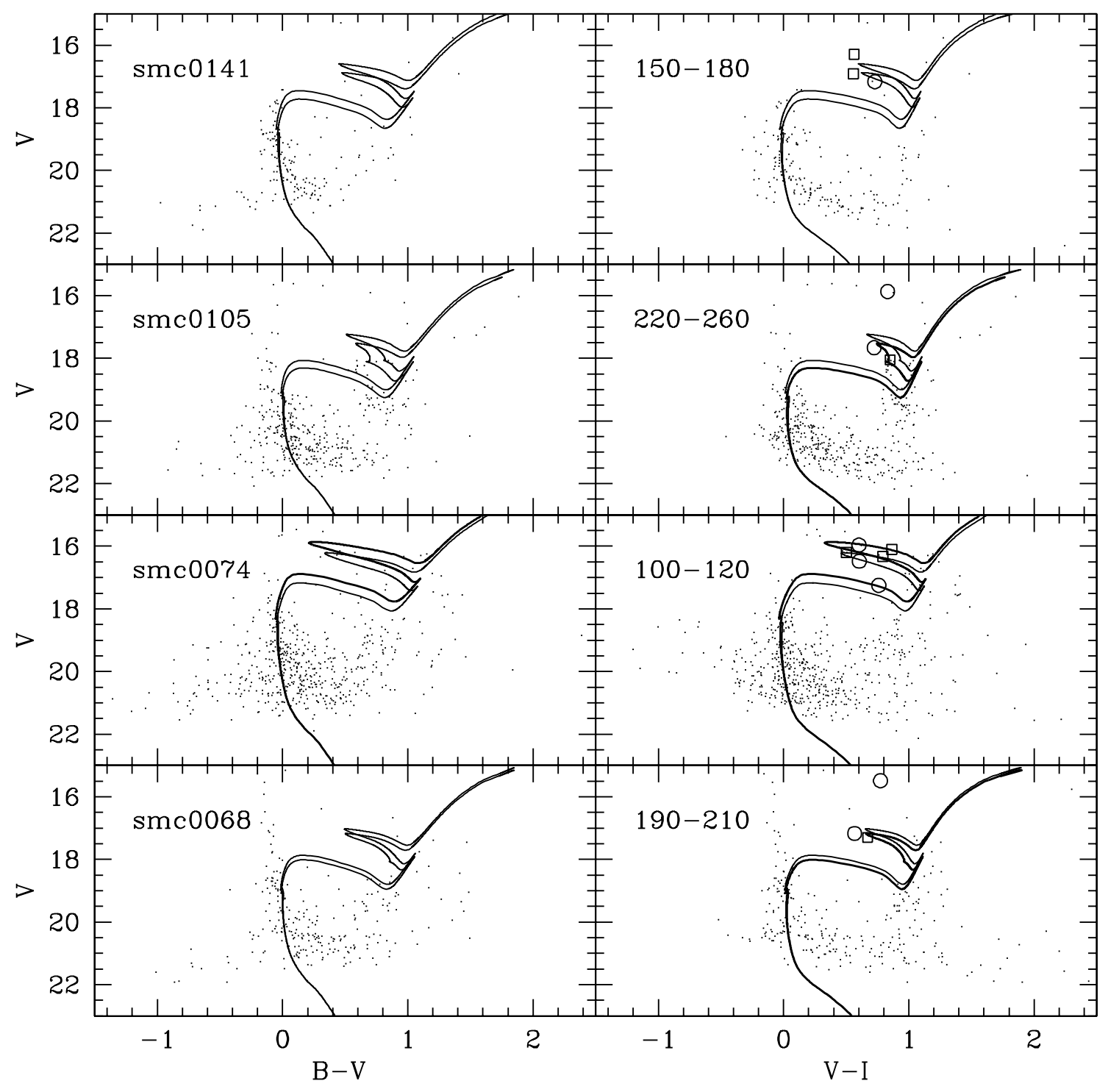

Fig. 7b.- Same as Fig. 7a but for a different sample of SMC clusters. 


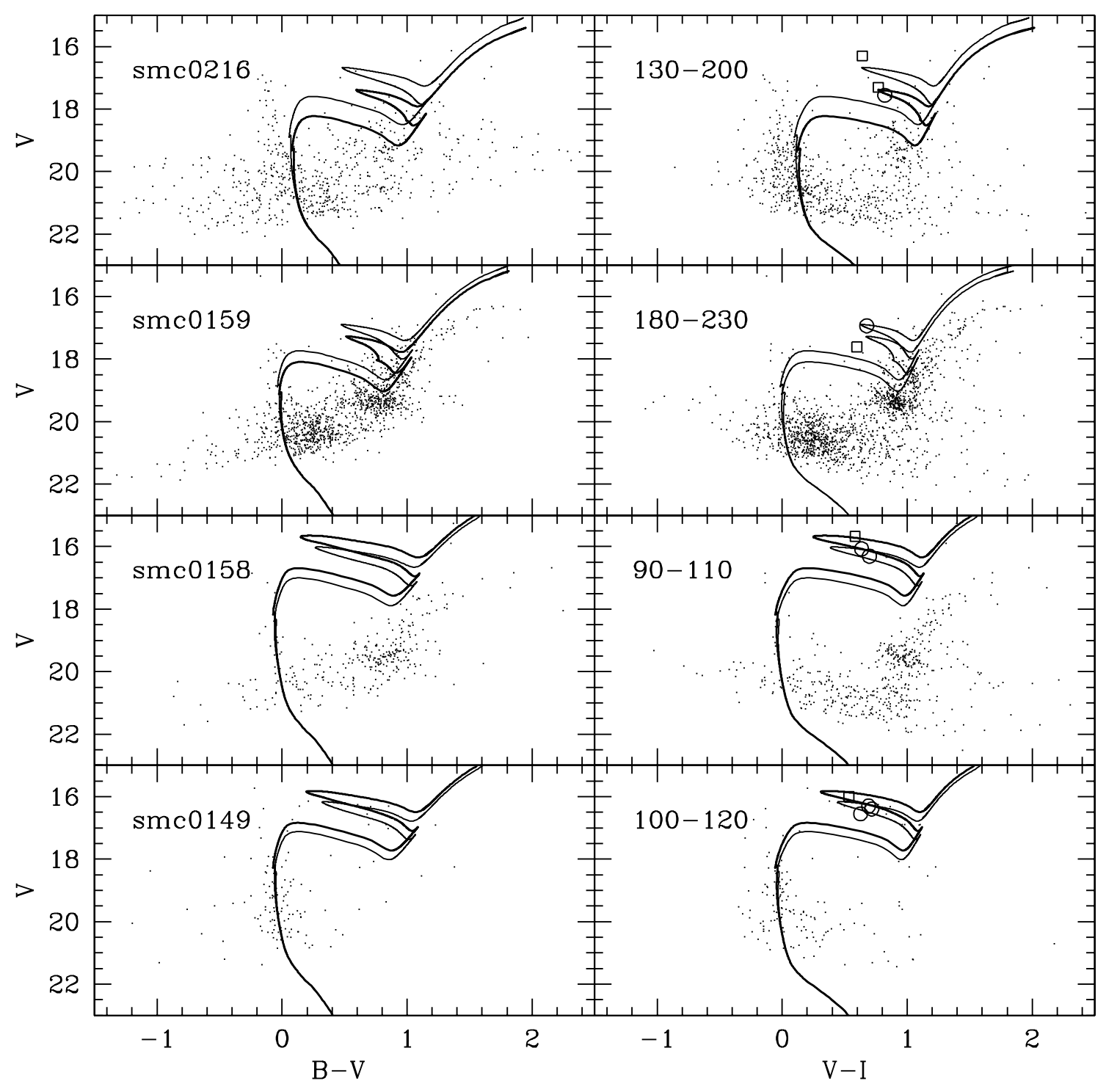

Fig. 7c.- Same as Fig. 7a but for a different sample of SMC clusters. 


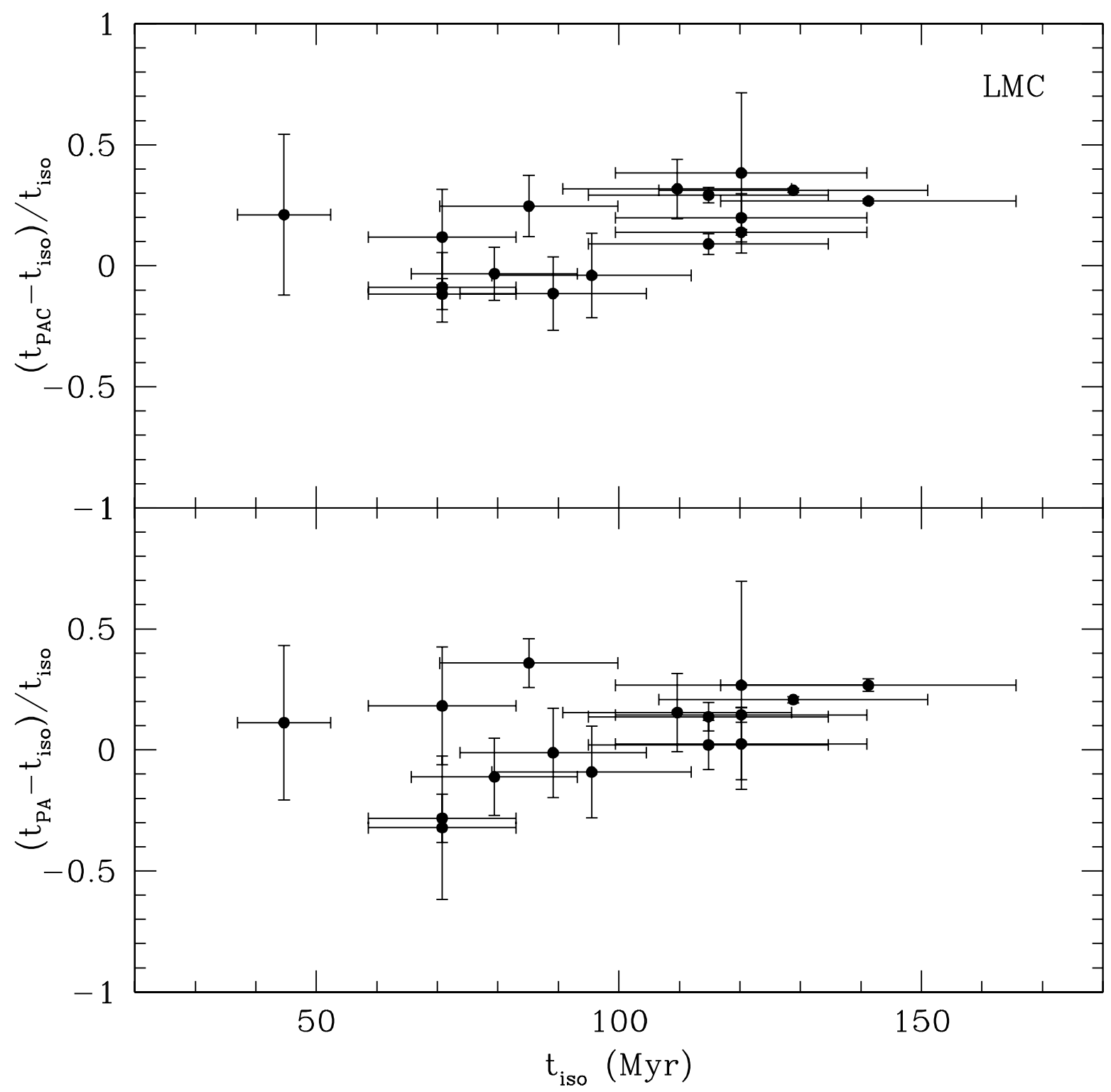

Fig. 8.- Relative difference between evolutionary and pulsation cluster ages as a function of isochrone ages for LMC clusters that host at least two Cepheids. The top panel shows the difference with the PAC relation, while the bottom one with the PA relation. 


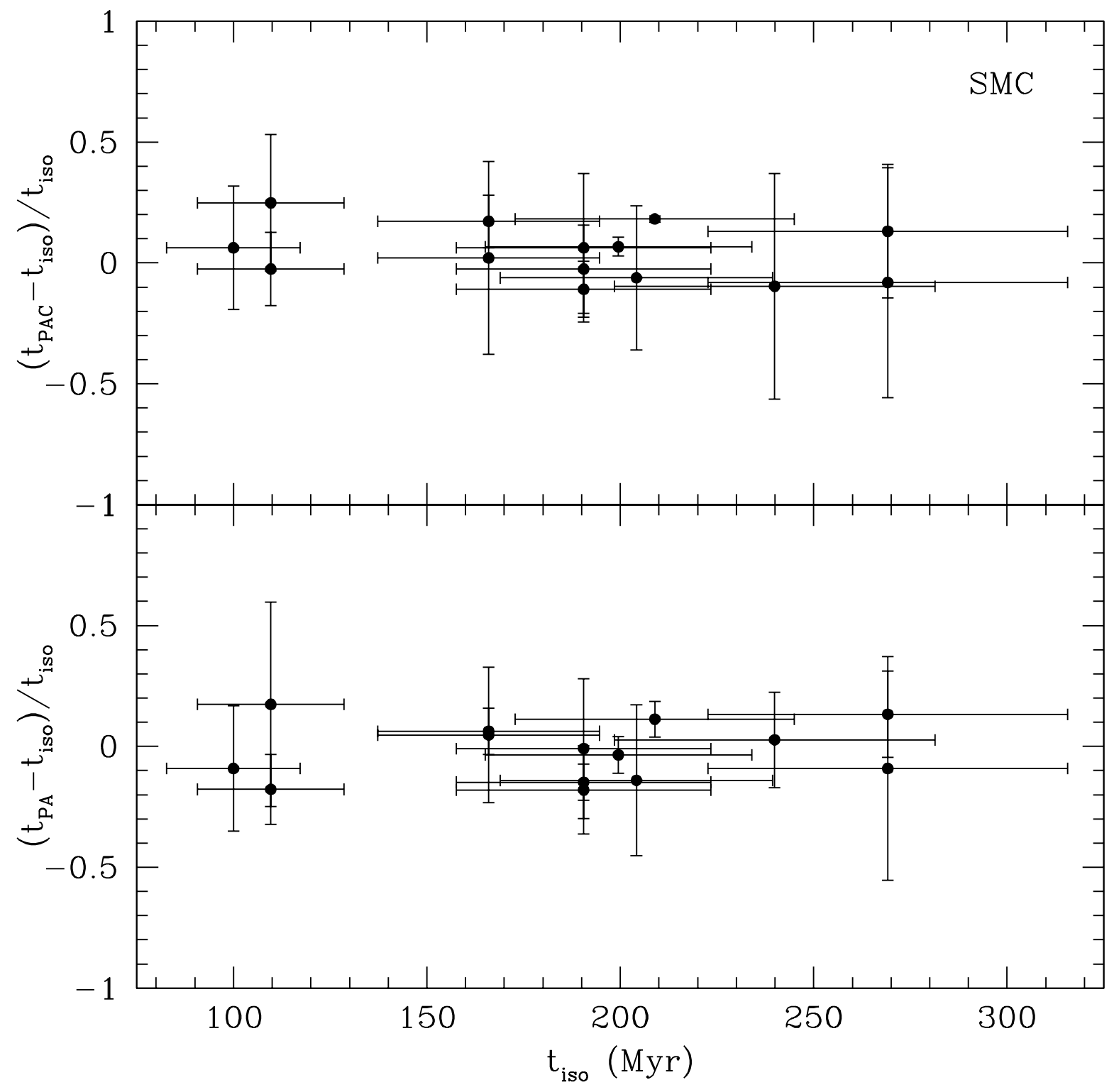

Fig. 9.- Same as Fig. 8, but for SMC clusters. See text for more details. 


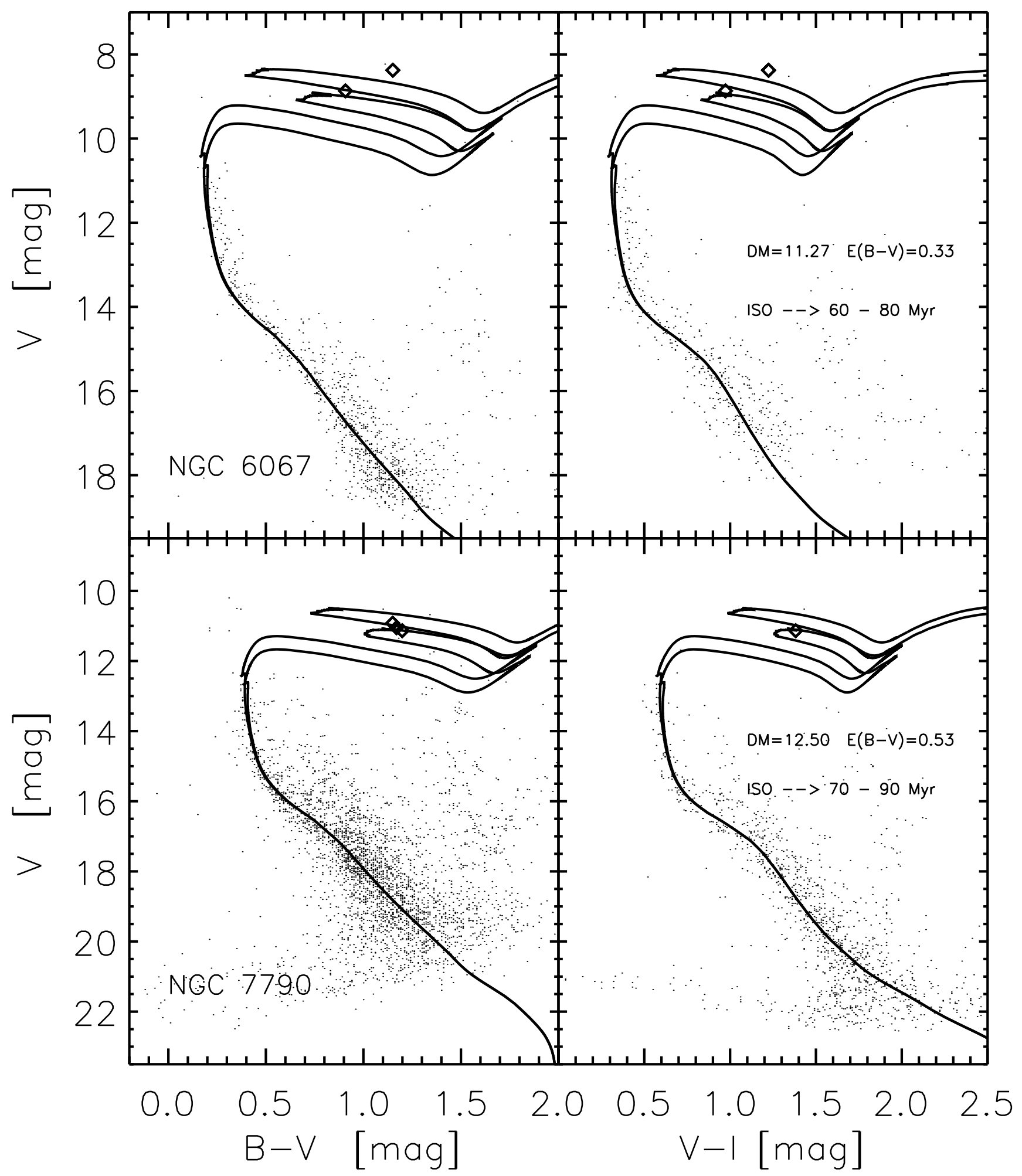

Fig. 10. - Same as Fig. 6a, but for two Galactic clusters and stellar isochrones with a metal content of $\mathrm{Z}=0.02$. 
Table 1. Input parameters of the pulsation models adopted to compute the PA and the $\mathrm{PAC}$ relations.

\begin{tabular}{|c|c|c|c|c|c|c|c|c|c|c|c|}
\hline $\begin{array}{l}M^{\mathrm{a}} \\
(1)\end{array}$ & $\begin{array}{c}\log L^{\mathrm{b}} \\
(2)\end{array}$ & $\begin{array}{l}M^{\mathrm{a}} \\
(3)\end{array}$ & $\begin{array}{c}\log L^{\mathrm{b}} \\
(4)\end{array}$ & $\begin{array}{l}M^{\mathrm{a}} \\
(5)\end{array}$ & $\begin{array}{c}\log L^{\mathrm{b}} \\
(6)\end{array}$ & $\begin{array}{l}M^{\mathrm{a}} \\
(7)\end{array}$ & $\begin{array}{c}\log L^{\mathrm{b}} \\
(8)\end{array}$ & $\begin{array}{l}M^{\mathrm{a}} \\
(9)\end{array}$ & $\begin{array}{c}\log L^{\mathrm{b}} \\
(10)\end{array}$ & $\begin{array}{c}M^{\mathrm{a}} \\
(11)\end{array}$ & $\begin{array}{c}\log L^{\mathrm{b}} \\
(12)\end{array}$ \\
\hline \multicolumn{2}{|c|}{$\mathrm{Z}=0.004^{\mathrm{c}}$} & \multicolumn{2}{|c|}{$\mathrm{Z}=0.008^{\mathrm{c}}$} & \multicolumn{2}{|c|}{$\mathrm{Z}=0.02^{\mathrm{c}}$} & \multicolumn{2}{|c|}{$\mathrm{Z}=0.004^{\mathrm{c}}$} & \multicolumn{2}{|c|}{$\mathrm{Z}=0.008^{\mathrm{c}}$} & \multicolumn{2}{|c|}{$\mathrm{Z}=0.02^{\mathrm{c}}$} \\
\hline \multicolumn{6}{|c|}{ First-overtone Canonical $^{\mathrm{d}}$} & \multicolumn{6}{|c|}{ First-overtone Noncanonical ${ }^{\mathrm{e}}$} \\
\hline 3.25 & 2.49 & 3.25 & 2.45 & 3.50 & 2.51 & 3.00 & 2.62 & 3.00 & 2.58 & 3.00 & 2.52 \\
\hline 3.50 & 2.61 & 3.50 & 2.47 & 4.00 & 2.72 & 3.50 & 2.86 & 3.50 & 2.82 & 4.00 & 2.97 \\
\hline 3.80 & 2.74 & 3.80 & 2.70 & 4.50 & 2.90 & 4.00 & 3.07 & 4.00 & 3.03 & 4.60 & 3.19 \\
\hline 4.00 & 2.82 & 4.00 & 2.78 & 5.00 & 3.07 & 5.00 & 3.30 & 5.00 & 3.30 & 4.75 & 3.24 \\
\hline 5.00 & 3.07 & 5.00 & 3.07 & 5.50 & 3.22 & 5.25 & 3.50 & 5.25 & 3.46 & . & $\ldots$ \\
\hline 5.50 & 3.32 & 7.00 & 3.65 & 5.60 & 3.25 & 5.50 & 3.57 & 5.50 & 3.53 & & $\ldots$ \\
\hline 5.80 & 3.40 & 7.30 & 3.72 & $\ldots$ & $\ldots$ & 5.60 & 3.60 & 5.60 & 3.55 & & $\ldots$ \\
\hline 7.00 & 3.65 & $\ldots$ & $\ldots$ & $\ldots$ & $\cdots$ & $\ldots$ & $\ldots$ & $\ldots$ & $\ldots$ & 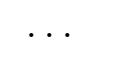 & $\cdots$ \\
\hline 7.15 & 3.73 & $\ldots$ & $\ldots$ & $\ldots$ & $\ldots$ & $\ldots$ & $\ldots$ & $\ldots$ & $\ldots$ & . & $\ldots$ \\
\hline 7.30 & 3.76 & $\ldots$ & $\ldots$ & $\ldots$ & $\ldots$ & $\ldots$ & $\ldots$ & $\ldots$ & $\ldots$ & $\ldots$ & $\ldots$ \\
\hline \multicolumn{6}{|c|}{ Fundamental Canonical $^{\mathrm{d}}$} & \multicolumn{6}{|c|}{ Fundamental Noncanonicale } \\
\hline 3.25 & 2.49 & 3.25 & 2.45 & 4.50 & 2.90 & 3.50 & 2.86 & 4.0 & 3.07 & 4.00 & 3.22 \\
\hline 3.50 & 2.61 & 3.50 & 2.57 & 5.00 & 3.07 & 5.00 & 3.30 & 5.00 & 3.30 & 5.00 & 3.30 \\
\hline 3.80 & 2.74 & 3.80 & 2.70 & 6.25 & 3.42 & 7.00 & 3.85 & 7.00 & 3.85 & 7.00 & 3.85 \\
\hline 4.00 & 2.82 & 4.00 & 2.78 & 6.50 & 3.48 & 9.00 & 4.25 & 9.00 & 4.25 & 9.00 & 4.25 \\
\hline 5.00 & 3.07 & 5.00 & 3.07 & 6.75 & 3.54 & 11.00 & 4.65 & 11.00 & 4.65 & 11.00 & 4.65 \\
\hline 7.00 & 3.65 & 6.55 & 3.55 & 7.00 & 3.65 & $\ldots$ & $\ldots$ & $\ldots$ & $\ldots$ & $\cdots$ & $\ldots$ \\
\hline 7.15 & 3.73 & 6.70 & 3.59 & 9.00 & 4.00 & $\ldots$ & $\ldots$ & $\ldots$ & $\ldots$ & $\ldots$ & $\ldots$ \\
\hline 7.30 & 3.76 & 6.85 & 3.62 & 11.00 & 4.40 & $\ldots$ & $\ldots$ & $\ldots$ & $\ldots$ & & $\ldots$ \\
\hline 7.45 & 3.79 & 7.00 & 3.65 & $\ldots$ & $\ldots$ & . & . & $\ldots$ & $\ldots$ & & $\ldots$ \\
\hline 9.00 & 4.00 & 7.15 & 3.69 & $\ldots$ & 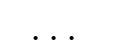 & . . & 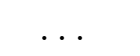 & $\ldots$ & . & & $\ldots$ \\
\hline \multirow[t]{4}{*}{11.00} & 4.40 & 7.30 & 3.72 & $\ldots$ & $\ldots$ & $\ldots$ & $\ldots$ & $\ldots$ & $\ldots$ & $\ldots$ & $\ldots$ \\
\hline & $\because$ & 7.45 & 3.75 & $\ldots$ & & & & $\ldots$ & $\ldots$ & & $\ldots$ \\
\hline & 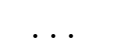 & 9.00 & 4.00 & $\ldots$ & & & & $\ldots$ & $\ldots$ & & $\ldots$ \\
\hline & & 11.00 & 4.40 & $\ldots$ & . & · & $\ldots$ & $\ldots$ & $\ldots$ & $\ldots$ & $\ldots$ \\
\hline
\end{tabular}

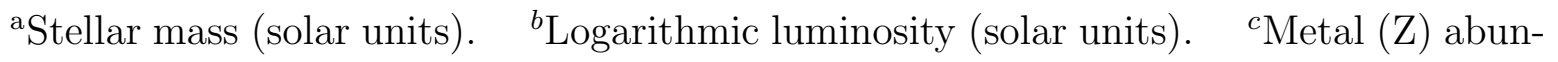
dances by mass; the helium abundances are $\mathrm{Y}=0.25(\mathrm{Z}=0.004, \mathrm{Z}=0.008)$ and $\mathrm{Y}=0.28$ $(\mathrm{Z}=0.02) . \quad{ }^{d}$ Pulsation models constructed by adopting a M/L relation based on evolutionary models that neglect the convective core overshooting (Bono et al. 1999).

${ }^{e}$ Pulsation models constructed by adopting a M/L relation that mimics the behavior of evolutionary models that account for the convective core overshooting during H-burning (Chiosi et al. 2003; Bono et al. 1999). 
Table 2. Analytical relations $\left(\log T_{e}=\alpha+\beta \log L / L_{\odot}\right)$ for the boundaries of the instability strip at different chemical compositions.

\begin{tabular}{|c|c|c|c|c|c|c|}
\hline $\begin{array}{c}Z^{\mathrm{a}} \\
(1)\end{array}$ & $\begin{array}{c}\alpha^{\mathrm{b}} \\
(2)\end{array}$ & $\begin{array}{l}\beta^{\mathrm{c}} \\
(3)\end{array}$ & $\begin{array}{c}\sigma^{\mathrm{d}} \\
(4)\end{array}$ & $\begin{array}{c}\alpha^{\mathrm{b}} \\
(5)\end{array}$ & $\begin{array}{l}\beta^{\mathrm{c}} \\
(6)\end{array}$ & $\begin{array}{c}\sigma^{\mathrm{d}} \\
(7)\end{array}$ \\
\hline & \multicolumn{3}{|c|}{ Canonical $^{\mathrm{e}}$} & \multicolumn{3}{|c|}{ Noncanonical $^{\mathrm{f}}$} \\
\hline & \multicolumn{6}{|c|}{ First-overtone blue edge } \\
\hline 0.004 & $3.933 \pm 0.008$ & $-0.041 \pm 0.005$ & 0.008 & $3.967 \pm 0.002$ & $-0.053 \pm 0.003$ & 0.002 \\
\hline 0.008 & $3.961 \pm 0.005$ & $-0.052 \pm 0.004$ & 0.005 & $3.959 \pm 0.002$ & $-0.052 \pm 0.002$ & 0.002 \\
\hline 0.02 & $3.974 \pm 0.009$ & $-0.061 \pm 0.014$ & 0.008 & .. & $\cdots$ & $\cdots$ \\
\hline & \multicolumn{6}{|c|}{ First-overtone red edge } \\
\hline 0.004 & $3.820 \pm 0.008$ & $-0.017 \pm 0.005$ & 0.007 & $3.869 \pm 0.011$ & $-0.031 \pm 0.012$ & 0.010 \\
\hline 0.008 & $3.831 \pm 0.006$ & $-0.021 \pm 0.005$ & 0.005 & $3.839 \pm 0.010$ & $-0.023 \pm 0.010$ & 0.009 \\
\hline 0.02 & $3.803 \pm 0.003$ & $-0.012 \pm 0.005$ & 0.003 & & & \\
\hline \multicolumn{7}{|c|}{ Fundamental blue edge } \\
\hline 0.004 & $3.812 \pm 0.007$ & $-0.012 \pm 0.003$ & 0.07 & $3.856 \pm 0.008$ & $-0.023 \pm 0.006$ & 0.007 \\
\hline 0.008 & $3.838 \pm 0.010$ & $-0.021 \pm 0.005$ & 0.010 & $3.907 \pm 0.009$ & $-0.040 \pm 0.007$ & 0.008 \\
\hline 0.02 & $3.949 \pm 0.007$ & $-0.059 \pm 0.006$ & 0.007 & $3.955 \pm 0.007$ & $-0.059 \pm 0.006$ & 0.007 \\
\hline $0.02^{\mathrm{g}}$ & $3.940 \pm 0.005$ & $-0.056 \pm 0.005$ & 0.005 & & & \\
\hline \multicolumn{7}{|c|}{ Fundamental red edge } \\
\hline 0.004 & $3.923 \pm 0.008$ & $-0.061 \pm 0.004$ & 0.008 & $3.957 \pm 0.007$ & $-0.072 \pm 0.006$ & 0.006 \\
\hline 0.008 & $3.947 \pm 0.008$ & $-0.070 \pm 0.004$ & 0.008 & $3.980 \pm 0.012$ & $-0.082 \pm 0.011$ & 0.010 \\
\hline 0.02 & $4.039 \pm 0.007$ & $-0.101 \pm 0.005$ & 0.007 & $4.118 \pm 0.014$ & $-0.122 \pm 0.012$ & 0.012 \\
\hline $0.02^{\mathrm{g}}$ & $4.061 \pm 0.009$ & $-0.110 \pm 0.008$ & 0.008 & & & $\ldots$ \\
\hline
\end{tabular}

${ }^{a}$ Metal abundance. $\quad{ }^{b}$ Zero Point. $\quad{ }^{c}$ Slope. $\quad d$ Standard deviations.

${ }^{e}$ Pulsation models constructed by adopting a $\mathrm{M} / \mathrm{L}$ relation based on evolutionary models that neglect the convective core overshooting (Bono et al. 1999).

${ }^{d}$ Pulsation models constructed by adopting a M/L relation that mimics the behavior of behavior of models that account for the convective core overshooting during H-burning (Chiosi et al. 2003; Bono et al. 1999). $\quad{ }^{g}$ Pulsation models from Petroni et al. (2003). 
Table 3. Analytical relations $\left(\log T_{e}=\alpha+\beta \log L / L_{\odot}+\gamma\left(\log L / L_{\odot}\right)^{2}\right)$ for the boundaries of the canonical fundamental instability strip at different chemical compositions.

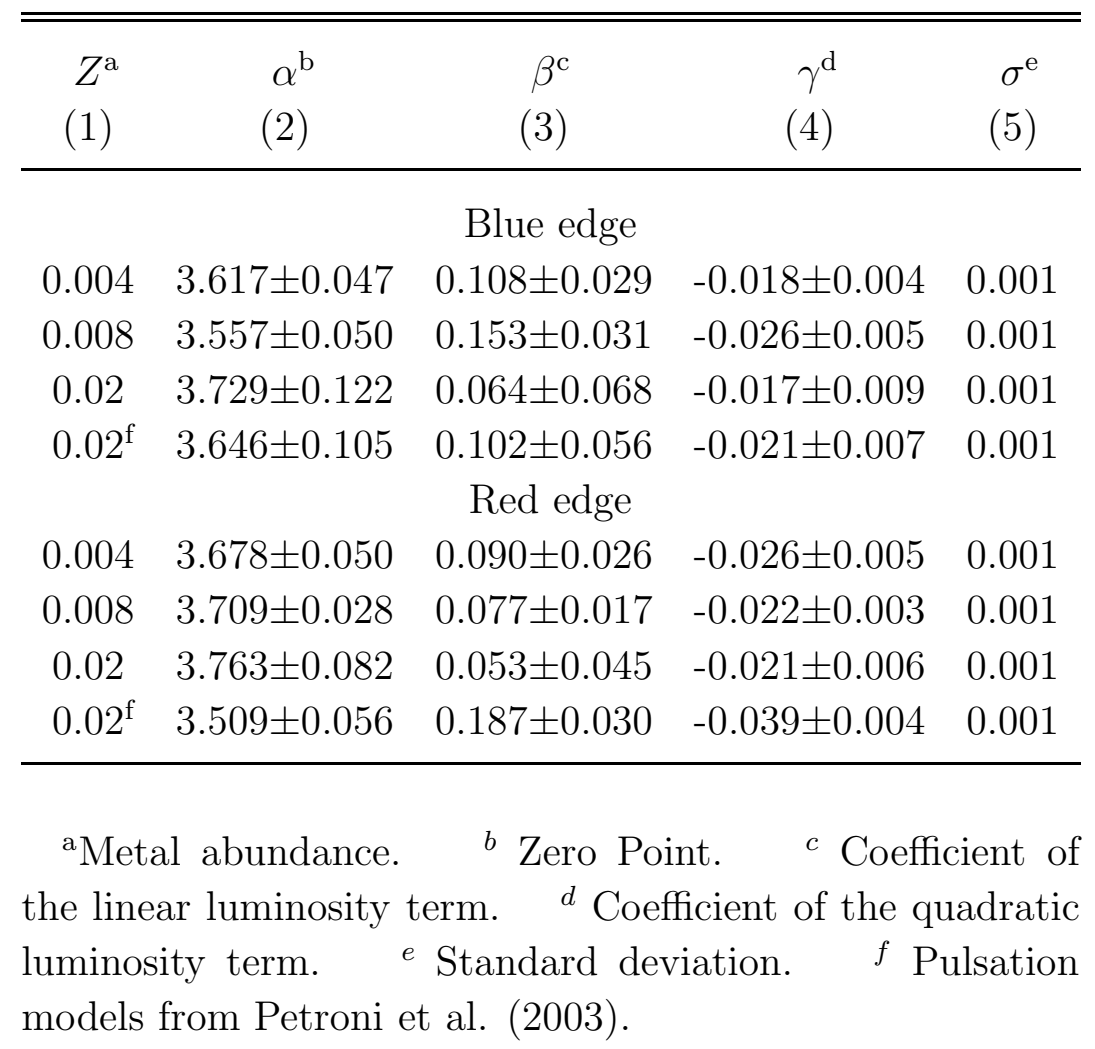


Table 4. Period-Age relations $\left(\log t^{\mathrm{a}}=\alpha+\beta \log P\right)$ at different chemical compositions.

\begin{tabular}{cccc}
\hline \hline $\begin{array}{c}Z^{\mathrm{b}} \\
(1)\end{array}$ & $\alpha^{\mathrm{c}}$ & $\beta^{\mathrm{d}}$ & $\sigma^{\mathrm{e}}$ \\
$(2)$ & $(3)$ & $(4)$ \\
\hline \multicolumn{4}{c}{ First-overtone } \\
0.004 & $8.41 \pm 0.07$ & $-1.07 \pm 0.02$ & 0.07 \\
0.01 & $8.29 \pm 0.08$ & $-0.80 \pm 0.03$ & 0.08 \\
0.02 & $8.08 \pm 0.04$ & $-0.39 \pm 0.04$ & 0.04 \\
\multicolumn{4}{c}{ Fundamental } \\
0.004 & $8.49 \pm 0.09$ & $-0.79 \pm 0.01$ & 0.09 \\
0.01 & $8.41 \pm 0.10$ & $-0.78 \pm 0.01$ & 0.10 \\
0.02 & $8.31 \pm 0.08$ & $-0.67 \pm 0.01$ & 0.08 \\
\hline \multicolumn{4}{c}{} \\
${ }^{\mathrm{a} C e p h e i d}$ & age in years. & ${ }^{b}$ Metal \\
abundance. & ${ }^{c}$ Zero Point. & ${ }^{d}$ Slope.
\end{tabular}

${ }^{e}$ Standard deviations. 
Table 5. Period and ages for selected evolutionary models.

\begin{tabular}{|c|c|c|c|c|c|c|c|c|}
\hline $\begin{array}{c}M / M_{\odot}{ }^{\mathrm{a}} \\
(1)\end{array}$ & $\begin{array}{c}\log L / L_{\odot}{ }^{\mathrm{b}} \\
\quad(2)\end{array}$ & $\begin{array}{c}\log T_{e}^{\mathrm{c}} \\
\quad(3)\end{array}$ & $\begin{array}{c}\log P^{\mathrm{d}} \\
(4)\end{array}$ & $\begin{array}{c}\operatorname{Age}_{I S O} \mathrm{e}^{\mathrm{e}} \\
\quad(5)\end{array}$ & $\begin{array}{c}\log L / L_{\odot}{ }^{\mathrm{b}} \\
(6)\end{array}$ & $\begin{array}{c}\log T_{e}^{\mathrm{c}} \\
\quad(7)\end{array}$ & $\begin{array}{c}\log P^{\mathrm{d}} \\
(8)\end{array}$ & $\begin{array}{c}\text { Age }_{I S O}{ }^{\mathrm{e}} \\
\quad(9)\end{array}$ \\
\hline \multicolumn{6}{|c|}{ Canonical } & \multirow{2}{*}{\multicolumn{2}{|c|}{ Noncanonical }} & \\
\hline \multicolumn{7}{|c|}{$\mathrm{Z}=0.004, \mathrm{Y}=0.251^{\mathrm{f}}$} & & \\
\hline 4 & 2.935 & 3.760 & 0.472 & 149.69 & 3.149 & 3.757 & 0.682 & 171.04 \\
\hline 5 & 3.249 & 3.749 & 0.724 & 85.65 & 3.419 & 3.744 & 0.897 & 94.35 \\
\hline 8 & 3.900 & 3.725 & 1.243 & 30.16 & 4.089 & 3.712 & 1.462 & 34.98 \\
\hline 10 & 4.159 & 3.716 & 1.437 & 19.16 & 4.382 & 3.698 & 1.702 & 22.96 \\
\hline \multicolumn{9}{|c|}{$\mathrm{Z}=0.008, \mathrm{Y}=0.256$} \\
\hline 4 & 2.751 & 3.767 & 0.287 & 142.73 & & & & \\
\hline 5 & 3.162 & 3.749 & 0.653 & 89.39 & 3.360 & 3.739 & 0.870 & 97.85 \\
\hline 8 & 3.879 & 3.716 & 1.265 & 32.36 & 4.049 & 3.697 & 1.488 & 34.86 \\
\hline 10 & 4.163 & 3.703 & 1.496 & 19.24 & 4.354 & 3.678 & 1.756 & 23.56 \\
\hline \multicolumn{9}{|c|}{$\mathrm{Z}=0.0198, \mathrm{Y}=0.273$} \\
\hline 5 & 2.967 & 3.757 & 0.457 & 100.92 & $\ldots$ & & . & \\
\hline 8 & 3.760 & 3.693 & 1.245 & 29.93 & 3.951 & 3.679 & 1.469 & 32.79 \\
\hline 10 & 4.073 & 3.668 & 1.543 & 18.89 & 4.296 & 3.648 & 1.817 & 21.63 \\
\hline
\end{tabular}

${ }^{a}$ Stellar mass (solar units). $\quad{ }^{b}$ Luminosity (solar units). $\quad{ }^{c}$ Effective temperature (K). $\quad{ }^{d}$ Period (days). $\quad{ }^{e}$ Stellar age (My). $\quad{ }^{f}$ Metal (Z) and helium (Y) abundances by mass adopted to construct evolutionary models. 
Table 6. Period-Age-Color relations $\left(\log t^{\mathrm{a}}=\alpha+\beta \log P+\gamma C I\right)$ at different chemical compositions.

\begin{tabular}{ccccc}
\hline \hline$Z^{\mathrm{b}}$ & $\alpha^{\mathrm{c}}$ & $\beta^{\mathrm{d}}$ & $\gamma^{\mathrm{e}}$ & $\sigma^{\mathrm{f}}$ \\
$(1)$ & $(2)$ & $(3)$ & $(4)$ & $(5)$ \\
\hline \multicolumn{5}{c}{ First-overtone } \\
0.004 & $18.68 \pm 0.06$ & $-1.184 \pm 0.02$ & $-2.70 \pm 0.33^{\mathrm{g}}$ & 0.06 \\
0.004 & $8.14 \pm 0.06$ & $-1.17 \pm 0.02$ & $0.65 \pm 0.08^{\mathrm{h}}$ & 0.06 \\
0.004 & $8.06 \pm 0.06$ & $-1.16 \pm 0.02$ & $0.64 \pm 0.08^{\mathrm{i}}$ & 0.06 \\
0.01 & $18.27 \pm 0.08$ & $-0.89 \pm 0.04$ & $-2.63 \pm 0.61^{\mathrm{g}}$ & 0.08 \\
0.01 & $8.04 \pm 0.08$ & $-0.89 \pm 0.04$ & $0.53 \pm 0.14^{\mathrm{h}}$ & 0.08 \\
0.01 & $7.94 \pm 0.08$ & $-0.88 \pm 0.03$ & $0.61 \pm 0.15^{\mathrm{i}}$ & 0.08 \\
0.02 & $14.15 \pm 0.04$ & $-0.48 \pm 0.05$ & $-1.60 \pm 0.60^{\mathrm{g}}$ & 0.04 \\
0.02 & $7.94 \pm 0.04$ & $-0.47 \pm 0.05$ & $0.31 \pm 0.12^{\mathrm{h}}$ & 0.04 \\
0.02 & $7.89 \pm 0.04$ & $-0.46 \pm 0.05$ & $0.36 \pm 0.14^{\mathrm{i}}$ & 0.04 \\
\multicolumn{5}{c}{ Fundamental } \\
0.004 & $14.65 \pm 0.09$ & $-0.88 \pm 0.02$ & $-1.63 \pm 0.36^{\mathrm{g}}$ & 0.09 \\
0.004 & $8.34 \pm 0.09$ & $-0.90 \pm 0.02$ & $0.35 \pm 0.06^{\mathrm{h}}$ & 0.09 \\
0.004 & $8.24 \pm 0.09$ & $-0.88 \pm 0.02$ & $0.42 \pm 0.08^{\mathrm{i}}$ & 0.09 \\
0.01 & $16.13 \pm 0.09$ & $-0.89 \pm 0.03$ & $-2.04 \pm 0.42^{\mathrm{g}}$ & 0.09 \\
0.01 & $8.24 \pm 0.09$ & $-0.90 \pm 0.03$ & $0.36 \pm 0.07^{\mathrm{h}}$ & 0.09 \\
0.01 & $8.13 \pm 0.09$ & $-0.89 \pm 0.03$ & $0.49 \pm 0.10^{\mathrm{i}}$ & 0.09 \\
0.02 & $11.60 \pm 0.08$ & $-0.75 \pm 0.03$ & $-0.87 \pm 0.34^{\mathrm{g}}$ & 0.08 \\
0.02 & $8.25 \pm 0.08$ & $-0.76 \pm 0.03$ & $0.16 \pm 0.06^{\mathrm{h}}$ & 0.08 \\
0.02 & $8.20 \pm 0.08$ & $-0.75 \pm 0.03$ & $0.21 \pm 0.08^{\mathrm{i}}$ & 0.08 \\
\hline
\end{tabular}

${ }^{a}$ Cepheid age in years. ${ }^{b}$ Metal abundance. $\quad{ }^{c}$ Zero Point. ${ }^{d}$ Coefficient of the period term. ${ }^{e}$ Coefficient of the color term. ${ }^{f}$ Standard deviations. ${ }^{g}$ The color term is $\log T_{e} . \quad{ }^{h}$ The color term is $B-V . \quad{ }^{i}$ The color term is $V-I$. 
Table 7. Age difference between canonical PA and PAC relations at selected periods.

\begin{tabular}{|c|c|c|c|c|c|c|c|c|c|}
\hline $\begin{array}{c}\log P^{\mathrm{a}} \\
(1)\end{array}$ & $\begin{array}{l}\mathrm{PA}^{\mathrm{b}} \\
(2)\end{array}$ & $\begin{array}{c}\operatorname{PAC}(\mathrm{BE})^{\mathrm{c}} \\
(3)\end{array}$ & $\begin{array}{c}\operatorname{PAC}(R E)^{\mathrm{d}} \\
(4)\end{array}$ & $\begin{array}{l}\mathrm{PA}^{\mathrm{b}} \\
(5)\end{array}$ & $\begin{array}{c}\mathrm{PAC}(\mathrm{BE})^{\mathrm{c}} \\
(6)\end{array}$ & $\begin{array}{c}\mathrm{PAC}(\mathrm{RE})^{\mathrm{d}} \\
(7)\end{array}$ & $\begin{array}{l}\mathrm{PA}^{\mathrm{b}} \\
(8)\end{array}$ & $\begin{array}{c}\operatorname{PAC}(\mathrm{BE})^{\mathrm{c}} \\
(9)\end{array}$ & $\begin{array}{c}\mathrm{PAC}(\mathrm{RE})^{\mathrm{d}} \\
(10)\end{array}$ \\
\hline & \multicolumn{3}{|c|}{$Z=0.004$} & \multicolumn{3}{|c|}{$Z=0.008$} & \multicolumn{3}{|c|}{$\mathrm{Z}=0.02$} \\
\hline 1 & 7.70 & $7.64(3.76)$ & $7.72(3.71)$ & 7.63 & $7.57(3.76)$ & $7.67(3.71)$ & 7.64 & $7.60(3.73)$ & $7.65(3.68)$ \\
\hline 1.5 & 7.30 & $7.23(3.74)$ & $7.33(3.68)$ & 7.24 & $7.21(3.72)$ & $7.30(3.67)$ & 7.30 & $7.27(3.68)$ & $7.31(3.64)$ \\
\hline
\end{tabular}

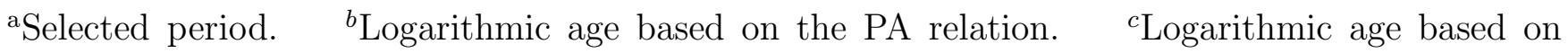
the PAC relation along the blue edge of the instability strip. The number in parentheses is the logarithmic temperature in which the period is equal to $\log P=1$, or $1.5 .{ }^{d}$ Logarithmic age based on the PAC relation along the red edge of the instability strip. The number in parentheses is the logarithmic temperature in which the period is equal to $\log P=1$, or 1.5 . 
Table 8. Age estimates for LMC and SMC open clusters that host at least two Cepheids.

\begin{tabular}{cccccccc}
\hline \hline $\begin{array}{c}\mathrm{ID} \\
(1)\end{array}$ & $N_{c}^{\mathrm{b}}$ & $E(B-V)^{\mathrm{c}}$ & $\log t_{I S O}^{\mathrm{d}}$ & $\log t_{P A}{ }^{\mathrm{e}}$ & $\sigma_{P A}^{\mathrm{f}}$ & $\log t_{P A C} \mathrm{~g}$ & $\sigma_{P A C} \mathrm{~h}$ \\
& $(2)$ & $(3)$ & $(4)$ & $(5)$ & $(6)$ & $(7)$ & $(8)$ \\
\hline LMC0394 & 2 & 0.138 & 8.15 & 8.015 & 0.015 & 8.015 & 0.005 \\
LMC0261 & 3 & 0.142 & 7.85 & 7.958 & 0.033 & 7.898 & 0.024 \\
LMC0164 & 4 & 0.144 & 8.08 & 7.945 & 0.255 & 7.870 & 0.233 \\
LMC0164 & 3 & 0.141 & 8.08 & 8.069 & 0.066 & 7.984 & 0.054 \\
LMC0142 & 3 & 0.152 & 7.65 & 7.598 & 0.156 & 7.547 & 0.183 \\
LMC0461 & 3 & 0.120 & 7.85 & 7.763 & 0.129 & 7.795 & 0.097 \\
LMC0457 & 7 & 0.120 & 7.93 & 7.737 & 0.068 & 7.807 & 0.073 \\
LMC0411 & 9 & 0.145 & 7.98 & 8.018 & 0.075 & 7.997 & 0.073 \\
LMC0395 & 2 & 0.138 & 8.08 & 8.012 & 0.015 & 8.015 & 0.005 \\
LMC0622 & 6 & 0.148 & 8.06 & 7.996 & 0.029 & 7.910 & 0.019 \\
LMC0591 & 2 & 0.147 & 8.11 & 8.009 & 0.006 & 7.948 & 0.004 \\
LMC0565 & 2 & 0.121 & 8.06 & 8.051 & 0.045 & 8.019 & 0.020 \\
LMC0559 & 3 & 0.121 & 7.90 & 7.946 & 0.062 & 7.914 & 0.046 \\
LMC0648 & 9 & 0.175 & 7.95 & 7.955 & 0.079 & 7.997 & 0.059 \\
LMC0636 & 6 & 0.185 & 7.85 & 7.971 & 0.097 & 7.887 & 0.057 \\
LMC0633 & 13 & 0.162 & 8.04 & 7.967 & 0.083 & 7.874 & 0.078 \\
SMC0048 & 3 & 0.101 & 8.43 & 8.468 & 0.184 & 8.464 & 0.191 \\
SMC0048 & 2 & 0.101 & 8.43 & 8.368 & 0.089 & 8.369 & 0.138 \\
SMC0045 & 3 & 0.094 & 8.32 & 8.268 & 0.036 & 8.233 & 0.007 \\
SMC0024 & 2 & 0.089 & 8.28 & 8.340 & 0.028 & 8.291 & 0.077 \\
SMC0016 & 5 & 0.089 & 8.28 & 8.284 & 0.124 & 8.252 & 0.142 \\
SMC0016 & 3 & 0.089 & 8.28 & 8.352 & 0.067 & 8.325 & 0.045 \\
SMC0141 & 3 & 0.079 & 8.22 & 8.192 & 0.044 & 8.138 & 0.057 \\
SMC0105 & 2 & 0.097 & 8.38 & 8.368 & 0.088 & 8.420 & 0.185 \\
SMC0074 & 5 & 0.094 & 8.04 & 7.957 & 0.222 & 7.916 & 0.163 \\
SMC0068 & 2 & 0.101 & 8.30 & 8.315 & 0.032 & 8.270 & 0.018 \\
SMC0216 & 3 & 0.194 & 8.22 & 8.199 & 0.128 & 8.211 & 0.177 \\
SMC0159 & 2 & 0.084 & 8.31 & 8.367 & 0.119 & 8.336 & 0.122 \\
SMC0158 & 3 & 0.084 & 8.00 & 8.038 & 0.103 & 7.972 & 0.118 \\
SMC0149 & 4 & 0.079 & 8.04 & 8.111 & 0.053 & 8.051 & 0.064 \\
& & & & & & &
\end{tabular}


Table 8-Continued

\begin{tabular}{cccccccc}
\hline \hline $\mathrm{ID}^{\mathrm{a}}$ & $N_{c}^{\mathrm{b}}$ & $E(B-V)^{\mathrm{c}}$ & $\log t_{I S O}{ }^{\mathrm{d}}$ & $\log t_{P A}{ }^{\mathrm{e}}$ & $\sigma_{P A}{ }^{\mathrm{f}}$ & $\log t_{P A C}{ }^{\mathrm{g}}$ & $\sigma_{P A C}{ }^{\mathrm{h}}$ \\
$(1)$ & $(2)$ & $(3)$ & $(4)$ & $(5)$ & $(6)$ & $(7)$ & $(8)$ \\
\hline
\end{tabular}

${ }^{a}$ Cluster identification according to Pietrzynski \& Udalski (1999).

${ }^{\mathrm{b}}$ Number of cluster Cepheids.

${ }^{\mathrm{c}}$ Mean cluster reddening based on individual cluster Cepheid reddenings (Pietrzynski \& Udalski 1999).

${ }^{\mathrm{d}}$ Cluster age based on stellar isochrones (yr). The uncertainty on this estimate is of the order of $\sigma_{I S O}=0.075$ dex.

eMean cluster age based on fundamental and first overtone PA relations (yr).

${ }^{\mathrm{f}}$ Standard deviation.

g Mean cluster age based on fundamental and first overtone PAC relations (yr).

${ }^{\mathrm{h}}$ Standard deviation. 
Table 9. Age estimates for two Galactic open clusters that host at least two Cepheids.

\begin{tabular}{|c|c|c|c|c|c|c|c|c|}
\hline $\begin{array}{l}\mathrm{ID}^{\mathrm{a}} \\
(1)\end{array}$ & $\begin{array}{c}\log P^{\mathrm{b}} \\
(2)\end{array}$ & $\begin{array}{c}<B>^{\mathrm{c}} \\
(3)\end{array}$ & $\begin{array}{c}<V>^{\mathrm{c}} \\
(4)\end{array}$ & $\begin{array}{c}<I>^{\mathrm{c}} \\
(5)\end{array}$ & $\begin{array}{c}E(B-V)^{\mathrm{d}} \\
\quad(6)\end{array}$ & $\begin{array}{l}\log t_{P A}{ }^{\mathrm{e}} \\
\quad(7)\end{array}$ & $\begin{array}{c}\log t_{P A C}^{(B-V) \mathrm{f}} \\
(8)\end{array}$ & $\begin{array}{c}\log t_{P A C}^{(V-I)_{\mathrm{g}}} \\
(9)\end{array}$ \\
\hline \multicolumn{9}{|c|}{ NGC $7790^{\mathrm{h}}$} \\
\hline CEa Cas & 0.711 & 12.070 & 10.920 & $\ldots$ & 0.562 & 7.834 & 7.788 & $\ldots$ \\
\hline CEb Cas & 0.651 & 12.220 & 11.050 & $\ldots$ & 0.548 & 7.874 & 7.827 & \\
\hline CF Cas & 0.688 & 12.335 & 11.136 & 9.754 & 0.531 & 7.849 & 7.824 & 7.838 \\
\hline \multicolumn{9}{|c|}{ NGC $6067^{\mathrm{h}}$} \\
\hline QZ Nor & 0.578 & 9.774 & 8.866 & 7.893 & 0.249 & 7.923 & 7.872 & 7.865 \\
\hline V340 Nor & 1.053 & 9.526 & 8.375 & 7.151 & 0.315 & 7.605 & 7.704 & 7.708 \\
\hline
\end{tabular}

${ }^{\text {a }}$ Cluster variable identification. $\quad{ }^{b}$ Logarithmic Period [days]. $\quad{ }^{c}$ Mean B, V,I magnitudes (mag). $\quad{ }^{d}$ Reddening. $\quad{ }^{e}$ Age estimate based on the PA relation (yr). ${ }^{f}$ Age estimate based on the $\mathrm{PAC}_{(B-V)}$ relation. ${ }^{g}$ Age estimate based on the $\mathrm{PAC}_{(V-I)}$ relation. ${ }^{h}$ Photometry and reddening estimates for cluster variables from Berdnikov (2000) [NGC 7790], and Laney \& Stobie (1994) [NGC 6067]. 